Fukunaga-Koontz Feature Transformation for Statistical Structural Damage Detection and Hierarchical Neuro-Fuzzy Damage Localisation

\author{
$\underline{\text { Simon Hoell }}$ \\ Institute of Structural Mechanics \\ Bauhaus University Weimar \\ 99423-Weimar \\ Germany
}

Piotr Omenzetter (corresponding author)

The LRF Centre for Safety and Reliability Engineering

The University of Aberdeen

Aberdeen, AB24 3UE

UK

Tel. 44-1224-272529

E-mail: piotr.omenzetter@abdn.ac.uk 


\begin{abstract}
Considering jointly damage sensitive features (DSFs) of signals recorded by multiple sensors, applying advanced transformations to these DSFs and assessing systematically their contribution to damage detectability and localisation can significantly enhance the performance of structural health monitoring systems. This philosophy is explored here for partial autocorrelation coefficients (PACCs) of acceleration responses. They are interrogated with the help of the linear discriminant analysis based on the Fukunaga-Koontz transformation using datasets of the healthy and selected reference damage states. Then, a simple but efficient fast forward selection procedure is applied to rank the DSF components with respect to statistical distance measures specialised for either damage detection or localisation. For the damage detection task, the optimal feature subsets are identified based on the statistical hypothesis testing. For damage localisation, a hierarchical neuro-fuzzy tool is developed that uses the DSF ranking to establish its own optimal architecture. The proposed approaches are evaluated experimentally on data from non-destructively simulated damage in a laboratory scale wind turbine blade. The results support our claim of being able to enhance damage detectability and localisation performance by transforming and optimally selecting DSFs. It is demonstrated that the optimally selected PACCs from multiple sensors or their Fukunaga-Koontz transformed versions can not only improve the detectability of damage via statistical hypothesis testing but also increase the accuracy of damage localisation when used as inputs into a hierarchical neuro-fuzzy network. Furthermore, the computational effort of employing these advanced soft computing models for damage localisation can be significantly reduced by using transformed DSFs.
\end{abstract}

Keywords: Damage detection, Damage localisation, Fukunaga-Koontz transformation, Neuro-fuzzy networks, Optimal feature selection, Statistical hypothesis testing, Wind turbines 


\section{LIST OF ACRONYMS}

ANFIS Adaptive neuro-fuzzy inference system

ANN Artificial neural network

AUC Area under curve

DSF Damage sensitive feature

FF $\quad$ Fast forward

FKT Fukunaga-Koontz transformation

HANFIS Hierarchical adaptive neuro-fuzzy inference systems

ICA Independent component analysis

LDA Linear discriminant analysis

LDA-FKT Linear discriminant analysis-based Fukunaga-Koontz transformation

LE Leading edge

PACC Partial autocorrelation coefficient

PACF Partial autocorrelation function

PCA Principal component analysis

SDD Structural damage detection

SDL Structural damage localization

SHM Structural health monitoring

$\mathrm{T} \quad$ Tip (of wind turbine blade)

TE Trailing edge

WT Wind turbine

WTB Wind turbine blade 


\section{INTRODUCTION}

The development of reliable, efficient and cost effective techniques for detecting and localising damage in structures continues to receive considerable attention across the aerospace, mechanical, structural, civil, energy, marine, electronic, electrical and other engineering disciplines. The driving factor is public and economic demand for reducing the life-cycle costs of existing and new structures and systems while guarantying their safe and reliable operations. Structural health monitoring (SHM) is an important technology for achieving these aims by providing information about the condition and performance of structures extracted from in-situ measurements [1]. Technologies originally developed for non-destructive testing, such as the X-radioscopy [2], thermal imaging [3] and eddy current methods [4], are often sensitive to changing in-situ environmental conditions and face challenges for continuous data collection in full-scale applications. Fibre optics strain measurements [5], acoustic emission [6] and active vibration techniques [7] have been tried by the SHM community for applications in large structures in harsh environments. Nevertheless, unless damage locations can be predetermined (as the so-called 'hot spots'), the essentially localised nature of those measurements will necessitate using dense sensor arrays, which entails high instrumentation and data analysis costs.

Passive vibration techniques for SHM utilize responses from ambient excitations. Environmental processes, such as wind, earthquakes or waves, and the operations itself, e.g. traffic on bridges or rotations of machinery, can excite global responses of large structures. Such vibrations are affected by structural damage, which typically takes the form of a change in mass, stiffness or energy dissipation mechanisms. They encompass the entire structure due to long wavelengths and low damping, thus, differences in the structural state can be observed with a small number of sensors. The downside is that some traditional vibration based signal features, e.g. the natural frequencies, may not offer high enough sensitivity to damage, or may suffer from excessive noise (e.g. mode shapes or their spatial derivatives) [810]. To overcome this and expand the range of usable damage sensitive features (DSFs) that can be extracted from vibration response signals, parametric time series model coefficients [11-15] and non-parametric time series representations in the frequency domain [16], timefrequency domain [17] or time domain [18] have been studied.

In addition to structural damage detection (SDD) [19], efficient structural damage localisation (SDL) is desired for delivering increased benefits in real-life SHM applications. SDL methods can be divided into local and global approaches. The local methods indicate 
damage locations by assessing the changes in signals of sensors placed at these locations. Kumar et al. [20] detected and located damage in a steel bridge using power spectral densities from different sensors. Various spectral functions were compared by Beskhyroun et al. [21] for SDL in experiments on a steel bridge. Similar to the previous approach, variations in DSFs from different sensors indicated damage location. Zheng and Mita [22] applied the cepstral and Itakura distances between autoregressive coefficients estimated from current and healthy state signals. Damage locations in numerical and experimental five-storey structures were identified by examining the distances for different sensors. In further studies, second order derivatives of fractal distances [23], distances between predictions of reconstructed attractor models [24] or deviations in symbolic time series DSFs from wavelet transformed signals [25] were proposed for SDL. Nevertheless, the SDL resolution of these approaches is limited by the number of sensors deployed and may therefore be poor on large and complex structures, and improvements could only be envisaged through significant investment in sensing hardware.

The global techniques may allow higher SDL resolutions by utilizing additional information about the physical properties of the monitored structure, its numerical model or known DSF characteristics for specific damage scenarios. Manson and Barthorpe [26] identified damages in different locations of an aircraft wing structure using DSFs composed of the damage-location-specific spectral lines of a frequency response function. Stochastic load vectors can be estimated from vibrational responses reflecting localised changes in structural flexibilities [27]. Using a numerical model of the healthy state structure, pseudo stresses can be computed and statistically tested for identifying damage locations [28]. Updating of numerical models with respect to experimentally determined characteristics, such as modal parameters, allows obtaining detailed damage information [29,30]. Nevertheless, selecting suitable characteristics often requires monitoring and controlling the process by the user or involves high computational demands during operation. Furthermore, the updating of numerical models often entails solving challenging ill-conditioned or deceptive inverse problems $[31,32]$.

The application of supervised soft computing techniques, characterised by acceptable requirements for computational resources and user interventions, presents an alternative for assessing the state of a structure. Worden et al. [33] prepared a comprehensive overview of soft computing techniques for mechanical systems with SHM applications also covered. Zhou et al. [34] applied the so-called random forest technique for damage classification in a four- 
storey steel frame benchmark structure and an experimental eight-storey steel shear frame structure, where DSFs were extracted with the help of wavelet packet decomposition of response accelerations. A support vector regression model was numerically trained for SDL in a small beam by Satpal et al. [35] with the first bending mode shape adopted as DSF. Using this model, damage was located experimentally with the help of a scanning laser Doppler vibrometer for estimating the mode shape. Offline post-damage classification of acousto-ultrasonic measurements in a pipework was performed by Tibaduiza et al. [36] by means of self-organizing maps. Osornio-Rios et al. [37] proposed the use of artificial neural networks (ANNs) for damage identification in combination with DSFs defined as amplitudes of eigenanalysis-based acceleration response spectra at selected natural frequencies. The method was applied to experiments on a five-bay truss-type structure under a sine sweep excitation. A review of the use of ANNs with DSFs based on frequency response functions in SHM was prepared by Hakim and Abdul Razak [38], where high DSF dimensionalities were identified as disadvantageous due to potential convergence problems and computational demands for optimizing the ANN parameters.

The challenges presented by the computational demands are common in soft computing networks but they can be alleviated by applying different dimensionality reduction techniques. The most straightforward way is to select only a subset of DSFs for further processing. The fast forward (FF) selection facilitated reduction of data for transmission in wireless sensor networks by consecutively adding wavelet transformation coefficients from Lamb wave signals to multivariate DSFs to maximize damage detection rates [39]. Zugasti et al. [40] applied a sequential FF selection for determining a subset of acceleration signals of a laboratory tower structure with the highest mutual information. From a comparison with principal component analysis (PCA) for feature extraction, the authors found that a combination of FF selection and PCA was beneficial for SDD. PCA allows identifying a linear orthogonal transformation which maximizes the variation in a dataset in the first few dimensions [41]. This property is commonly employed for removal of operational and environmental effects [42,43], dimensionality reduction [44], and extraction of features with improved sensitivities to damage [45]. Independent component analysis (ICA) is a more advanced linear technique, which tries to obtain statistically independent latent variables by accounting for higher order statistics. Hernandez-Garcia and Masri [46] compared PCA, ICA and modified ICA, where transformed variables were additionally required to be orthogonal, 
for sensor fault identification in vibration-based SHM systems, however, they did not observe a significant difference in performance.

Non-linear transformations can be effective when non-linear dependencies are present in the data. Torres Arredondo et al. [47] demonstrated an application of the hierarchical nonlinear PCA using an ANNs for SDD in a composite plate and a pipe work under temperature variations. Kernel PCA is another non-linear extension, where input data is non-linearly transformed to higher dimensional spaces with the help of non-linear kernel functions. The ratio between Mahalanobis distances of kernel PCA eigenvalues was proposed as damage index by Liu and Chattopadhyay [48] for SDD in a sandwich composite wing. Further dimensionality reduction techniques applied in SHM include local linear embedding [49] and feature assimilation [50]. However, the reviewed approaches did not systematically optimise the trade-off between dimensionality reduction and damage sensitivity of the resulting transformed DSFs.

The cornerstone of the approaches proposed in this paper is that performing advanced SHM tasks, such as SDD and SDL with global vibration methods, requires employing transformed DSFs which offer simultaneously an enhanced damage presence and/or location sensitivity as well as improved computational efficiency. Using features that are noisy or have poor sensitivity to damage presence or location can diminish the ability to distinguish between different structural states, especially for early damages where differences in DSFs are small. The present paper discusses transformations and selections of multivariate DSFs that aim at enhancing the distinguishability between damage states or locations while DSF dimensionalities are concurrently reduced. It cannot generally be guaranteed that DSFs and/or algorithms that perform optimally at the task of SDD will be equally accomplished when it comes to SDL, and vice versa. The transformation and selection of DSFs are therefore specialised in this research to allow the creation of dedicated statistical tests and neuro-fuzzy models for accurate SDD and SDL, respectively. The identification of such enhanced DSFs requires availability of data from the healthy state as well as selected damage states, thus the proposed methodologies for SDD and SDL can be understood as supervised. Nevertheless, different approaches can be utilized for obtaining the data, such as experiments or numerical simulations of the system in selected structural states and representative operational conditions, where practical considerations will often determine the choice.

Two approaches can be employed for generating training data, namely physical fullscale experiments and numerical physics-based simulations. Physical experiments are most 
suitable for creating databases of the healthy structure in realistic environmental and operational conditions. This can be done as part of post-construction inspections or using data from an SHM system installed during the initial post-construction period where it can be assumed that the structure is still in the healthy condition. It is fortunate that most wind turbines (WTs) will experience a wide range of operating and environmental conditions within relatively short time allowing for quick creation of adequate healthy datasets. For damage training datasets, numerical simulations will need to be relied on as they allow studying various damage scenarios by modifying healthy WT models with damages in different locations and with varying severities. Physics-based models can be further enhanced and calibrated with the already available SHM data from the healthy state, and environmental and operational conditions can also be included. The simulation of representative excitations will be crucial to assure the applicability to real structures. However, advanced models and tools are available, e.g. using transient dynamic simulations in finite element solvers, and they may only need fine tuning for specific conditions, which could again be done with available experimental data of the healthy WT. Thus, a combination of experiments and simulations will allow generating training data for the creation of SDD and SDL tools.

The linear discriminant analysis based on the Fukunaga-Koontz transformation (LDA-FKT) is introduced in this paper into the world of SHM. The original FukunagaKoontz transformation (FKT) is an extension of PCA developed initially for increasing the separability between two classes [51]. The FKT analyses jointly data from both classes and finds the best low-rank linear approximation of a quadratic classifier for these classes [52]. Solving a two-class problem is sufficient for SDD understood as distinguishing between the healthy and a damage structure without investigating which of the possible damage states occurred. Nevertheless, being able to distinguish between two classes only is often not sufficient for SDL because several structural states may occur and need to be considered for optimal performance of an SHM method. Therefore, the LDA-FKT is employed in this study. It allows handling multi-class problems by using between-class and within-class scatter matrices rather than variance-covariance matrices as was the case for the original FKT [53]. The scatter matrices can be computed using DSF samples from distinct structural states and the resulting transformation used to discriminate between these states.

However, a structural state with a given damage location can also have varying damage extents. Accordingly, the DSFs may not only vary with the location but also with the extent of damage. While we have opted for the simple statistical hypothesis testing for SDD, 
a further step was required to make the SDL process insensitive to the damage extent. (While it is possible, of course, to set off on a course to develop a scheme that also quantifies damage extent, this is outwith the scope of our present study.) Herein, adaptive neuro-fuzzy inference systems (ANFISes) are proposed for this task. An ANFIS is a Takagi-Sugeno fuzzy inference system, which can be represented as an ANN with a specific five-layer structure [54]. Its parameters can be optimized with the help of ANN training methods. Fuzzy inference systems create nonlinear mappings from inputs to outputs via fuzzy if-then rules. The structure of an ANFIS is prescribed by these rules, which are related to cluster analysis [55]. This allows identifying an appropriate structure in a systematic way, which is an advantage over other artificial or soft computing networks. Lei et al. [56] diagnosed faults in rotating machinery using multiple ANFISes, but each one was restricted to only four inputs of manually pre-selected DSFs. This was done to reduce the computational burden arising from grid-partitioning of the input space. To alleviate the so-called curse of dimensionality of neuro-fuzzy systems, using only relevant features as inputs and/or modifying the ANFIS structure has also been proposed [57].

A hierarchical ANFIS (HANFIS) modelling scheme is developed in this paper, which can account for differently distributed damage sensitivities in multivariate DSFs. First, the DSFs are ranked according to their ability to maximize an inter-class statistical distance using the FF approach. Then, the ranking is utilised while building the HANFIS by incrementally adding hierarchical levels with inputs of decreasing sensitivity to damage location. The optimal number of inputs is determined by presenting validation datasets to each model. This follows our central guiding principle that using only inputs with the highest sensitivities can lead to optimal performance, while over-fitting is prevented and computational efforts are reduced due to the lower number of inputs. The methodology proposed in this paper uses partial autocorrelation coefficients (PACCs) estimated from experimental acceleration signals as initial DSFs. PACCs are non-parametric time series representations with a close relationship to autocorrelation functions and parametric time series models [58]. PACCs from different sensors are studied separately and are then combined to explore the potential of multiple sensor DSF fusion for increased accuracy. For evaluating the proposed methodology, its performance in SDD using a statistical hypothesis test, and in SDL using HANFISes, is compared employing either the ranked initial DSFs or their LDA-FKT scores.

This methodology is studied using data from laboratory experiments with a smallscale wind turbine blade (WTB) made of a glass-fibre reinforced composite material. Wind- 
like excitations are applied with the help of a domestic pedestal fan. Damage is introduced non-destructively by attaching small masses at selected locations on the WTB's surface, where different masses simulate distinct damage extents. A WTB is selected for the method demonstration because vibration-based SHM in wind energy infrastructures is expected to be more critical, promising and beneficial compared to other applications. This is so because of the recent international commitments for achieving carbon neutrality by the end of this century [59]. The resulting increasing demands for wind energy lead to erections of ever larger WTs in locations, such as offshore, where winds are stronger and more predictable. However, these new developments in harsh environments are adversely affected by increasing operation and maintenance expenditures, which can make up to $20 \%$ of the total cost of energy production [60]. Consequently, the expected revenues and energy production targets are challenged. Adopting effective SHM techniques in WTBs may help to alleviate this undesirable situation.

The paper is structured as follows. The first section presents the general methodology we adopt for SDD and SDL and the corresponding theoretical background. The key aspects of the method working are explained using a theoretical illustrative example employing bivariate DSF for easy visualisation. In the following section, the experimental configuration and dynamic experiments with the small scale WTB are described. Then, the performance of the developed SDD and SDL procedures is investigated using experimental data, beginning with the estimation of initial DSFs, i.e. PACCs, then extraction of the LDA-FKT scores, selection of the optimal DSF subset for SDD, and finally comparison of the performance of the two DSF types for SDD. The identification and training of hierarchical neuro-fuzzy models for SDL using the selected and ranked initial and transformed DSFs is presented next, where the different hierarchical neuro-fuzzy models and DSF types are compared for their damage localisation accuracy. Finally, a summary and conclusions round up the paper.

\section{METHODOLOGY AND THEORY}

The steps of the proposed methodology for vibration-based SDD and SDL are shown in Fig. 1. It comprises two phases: an offline baseline or training phase and an online operational phase where damage is detected and localized. In the offline phase, the training of the algorithms is performed using acceleration datasets acquired in selected structural states. Since supervised algorithms perform best on scenarios similar to those on which they have been trained, the selection of training damage scenarios should be based on optimising the 
performance for the desired target SHM tasks. One practical way to select important scenarios is by considering the likelihood of damage occurrence and its consequences for the structural integrity, i.e. risk. To improve generalization capabilities of the method and its robustness, not only data from several locations is used but varying damage extents are considered as well (note our objectives are restricted to SDD and SDL, and so varying additionally damage extents is a potential threat to the SDD and SDL performance). Then, the dynamic response signals are pre-processed by filtering and resampling to reduce noise, dividing them into segments of a common length, and finally normalizing the segments by removing their estimated means and dividing by standard deviations to eliminate potential variations due to operational and environmental effects. In the next step, PACCs for each structural state considered are estimated up to the preselected numbers of lags.

The baseline phase then forks into two branches to train dedicated algorithms specialised for either SDD or SDL, respectively. In the SDD branch, all the available datasets (i.e. from the healthy and damaged states) are used to transform DSFs by means of the LDAFKT. Then, the FF selection algorithm is applied to the original PACCs and the resulting LDA-FKT scores using a statistical measure to describe their sensitivity to damage presence. This approach allows identifying the best DSF type and its optimal subset for binary statistical hypothesis testing to be applied for SDD (i.e. distinguishing between the healthy or damaged state) in the operational stage. Additionally, the statistical characteristics of these optimal DSFs for the healthy state, i.e. the mean vector and the variance-covariance matrix, are estimated from the training dataset and are also used in the operational phase.

The second branch aims at obtaining an optimal classifier for SDL, which will only be used in the operational phase when damage has been detected. Therefore, only datasets from damage states are used for training. Using these DSF samples, the LDA-FKT is performed and the resulting scores are ranked for their SDL performance with the help of a statistical measure for the multiclass problem. Similar ranking is performed for the original PACCs. The ranking information is used for creating HANFIS models for each DSF type with increasing numbers of inputs beginning with the most damage location sensitive features. An optimal HANFIS is identified by evaluating the error for cross-validation datasets. This completes the training of the algorithms, where the proposed offline procedure is beneficial because it allows utilizing advanced computational resources. Note that neither the DSF type nor its optimal subset need be the same for both SDD and SDL; indeed, it will be 
demonstrated later they are different for the theoretical illustrative example and WTB experimental example considered in this study.

In contrast to the baseline phase, the subsequent online operational phase entails only low computational effort. After pre-processing of signals of the structure in its current state, PACCs are estimated. If it is indicated as beneficial in the training phase, the PACCs are transformed into the selected LDA-FKT scores for SDD. Then, either the selected optimal PACCs or LDA-FKT scores are used for SDD by means of statistical hypothesis testing. If damage presence is indicated and the baseline phase established that PACCs should be transformed into LDA-FKT scores for SDL, this is done with the help of another LDA-FKT matrix built specifically for the SDL task. The highest ranked PACCs or LDA-FKT scores are then fed into the trained HANFIS to predict the location of the previously identified damage.

The following sections describe the theoretical tools used in the methodology: i) signal pre-processing and estimation of PACCs for the definition of initial DSFs, ii) the LDA-FKT, iii) statistical hypothesis testing and the FF algorithm for ranking of univariate DSF components with respect to their sensitivity to damage presence and localisation, and iv) the hierarchical neuro-fuzzy modelling.

\subsection{Partial autocorrelation-based damage sensitive features}

Measured vibration response time histories can be divided into $n$ segments with a common number of samples, $n_{\text {samp }}$. By removing the estimated means and dividing by the estimated standard deviations of each segment, zero-mean, unit-variance and normally distributed time series segments, $z_{i}[t]\left(i=1,2, \ldots, n ; t=1,2, \ldots, n_{\text {samp }}\right)$, can be obtained. A parsimonious representation of the signals is desired for efficient decision making about the current structural state. The partial autocorrelation function (PACF), $\alpha_{i}[\tau]$, is a non-parametric time series representation. It is the conditional correlation, $\operatorname{Corr}((\ldots) \mid(\ldots))$, between the time series $z_{i}[t]$ and its own version shifted by $\tau$ periods, $z_{i}[t-\tau]$, defined as $[61]$ :

$$
\alpha_{i}[\tau]=\operatorname{Corr}\left(z_{i}[t], z_{i}[t-\tau] \mid z_{i}[t-1], z_{i}[t-2], \ldots, z_{i}[t-\tau+1]\right)
$$

Thus, the effects of the intermediate variables, $z_{i}[t-1], z_{i}[t-2], \ldots, z_{i}[t-\tau+1]$, are removed.

Sample PACCs, $\hat{\alpha}_{i}[\tau]$, can efficiently be computed by the following recursion [58]:

$$
\hat{\alpha}_{i}[\tau]=\left(\hat{r}_{i}[\tau]-\sum_{p=1}^{\tau-1} \hat{a}_{i, p, \tau-1} \hat{r}_{i}[\tau-p]\right) /\left(1-\sum_{p=1}^{\tau-1} \hat{a}_{i, p, \tau-1} \hat{r}_{i}[p]\right)
$$


where $\hat{a}_{i, p, k}$ is the estimated $k$-th coefficient of an autoregressive model of order $p$ and $\hat{r}_{i}[\tau]$ is the sample autocorrelation coefficient at lag $\tau$ of the $i$-th time series segment. The close relationship between PACFs and autoregressive processes, as emphasised by the above formula, is commonly used for identifying autoregressive model orders to represent appropriately the underlying dynamics of such a process [62]. For lags higher than the required order, the PACF is theoretically zero and the PACCs follow approximately a zeromean Gaussian distribution with a standard deviation calculated as [63]:

$$
\sigma_{\hat{\alpha}_{i}[\tau]}=\sqrt{1 / n_{\text {samp }}} \text { for } \tau \geq p+1
$$

The estimated standard deviation enables selecting the significant PACCs as initial DSFs using elementary statistical tests [64].

Nevertheless, more information about the probability density functions of the PACCs is desired for performing statistic-based DSF ranking. A difficulty arises here because statistical distributions of PACCs are bounded between -1 and +1 and do not have obvious theoretical expressions. Therefore, Fisher's z-transformation is often applied for obtaining normally distributed z-scores, $\hat{\alpha}_{i}^{Z}[\tau]$, from PACCs [65]:

$$
\hat{\alpha}_{i}^{z}[\tau]=\tanh ^{-1}\left(\hat{\alpha}_{i}[\tau]\right)=\ln \left[\left(1+\hat{\alpha}_{i}[\tau]\right) /\left(1-\hat{\alpha}_{i}[\tau]\right)\right] / 2
$$

A multivariate DSF vector, $\mathbf{v}_{i}^{P A C}$, can then be composed of $m$ such z-scores as:

$$
\mathbf{v}_{i}^{P A C}=\left[\begin{array}{llll}
\hat{\alpha}_{i}^{z}[1] & \hat{\alpha}_{i}^{z}[2] & \cdots & \hat{\alpha}_{i}^{z}[m]
\end{array}\right]^{T} \sim \mathrm{N}(\boldsymbol{\mu}, \boldsymbol{\Sigma})
$$

where superscript $T$ denotes transpose. Due to the Fisher z-transformation, $\mathbf{v}_{i}^{P A C}$ follow a multivariate Gaussian probability distribution, $\mathrm{N}(\boldsymbol{\mu}, \boldsymbol{\Sigma})$, with a mean vector $\boldsymbol{\mu}$ and variancecovariance matrix $\boldsymbol{\Sigma}$. A DSF matrix, $\mathbf{V}^{P A C}$, can be constructed using DSF vectors from $n$ time series segments as follows:

$$
\mathbf{V}^{P A C}=\left[\begin{array}{llll}
\mathbf{v}_{1}^{P A C} & \mathbf{v}_{2}^{P A C} & \cdots & \mathbf{v}_{n}^{P A C}
\end{array}\right]
$$

\subsection{Linear discriminant analysis based on the Fukunaga-Koontz transformation}

In contrast to the well-known PCA or Karhunen-Loéve expansion, which aim at obtaining optimal approximations for reconstructing datasets from scores with reduced dimensionality, the FKT was developed for extracting low dimensional scores with improved classification capabilities in comparison to the initial features [51]. Huo [52] demonstrated theoretically that the FKT is the optimal linear approximation of a quadratic classifier for the two-class 
problem. However, for the applications in SHM, where a structure can be in one of several different states varying in, e.g., damage location and severity, the two-class formulation is insufficient. To circumvent this restriction, the use of LDA-FKT is proposed in this study. Multi-class LDA, also known as the Fisher discriminant analysis, tries to identify a linear transformation matrix, $\mathbf{W}$, which maximizes a function, $J_{F}(\mathbf{W})$, of the ratio between the class separation and the class overlap defined as [66]:

$$
J_{F}(\mathbf{W})=\operatorname{trace}\left[\left(\mathbf{W}^{T} \mathbf{S}_{w} \mathbf{W}\right)^{-1}\left(\mathbf{W}^{T} \mathbf{S}_{b} \mathbf{W}\right)\right]
$$

The distance between $C$ classes is quantified with the help of the between-class scatter matrix, $\mathbf{S}_{b}$, defined as:

$$
\mathbf{S}_{b}=\sum_{i=1}^{C} n_{i}\left(\hat{\boldsymbol{\mu}}_{i}-\hat{\boldsymbol{\mu}}_{T}\right)\left(\hat{\boldsymbol{\mu}}_{i}-\hat{\boldsymbol{\mu}}_{T}\right)^{T}
$$

where $n_{i}$ and $\widehat{\boldsymbol{\mu}}_{i}$ are the number of DSF samples and the estimated DSF mean vector of the $i$ th class, respectively. The mean, $\widehat{\boldsymbol{\mu}}_{T}$, of the total dataset containing $n_{T}$ DSF samples can be estimated as:

$$
\hat{\boldsymbol{\mu}}_{T}=\frac{1}{n_{T}} \sum_{i=1}^{C} n_{i} \hat{\boldsymbol{\mu}}_{i}
$$

The within-class scatter matrix, $\mathbf{S}_{w}$, for our chosen DSFs is defined as:

$$
\mathbf{S}_{w}=\sum_{j=1}^{C} \sum_{i=1}^{n_{j}}\left(\mathbf{v}_{i, j}^{P A C}-\hat{\boldsymbol{\mu}}_{i}\right)\left(\mathbf{v}_{i, j}^{P A C}-\hat{\boldsymbol{\mu}}_{i}\right)^{T}
$$

where the second index, $j$, indicates class belonging. A solution maximising the Fisher criterion of Eq. (7) can be found by solving the following generalized eigendecomposition problem [67]:

$$
\mathbf{S}_{w}^{-1} \mathbf{S}_{b} \mathbf{w}=\lambda \mathbf{w}
$$

where $\lambda$ is an eigenvalue and $\mathbf{w}$ is the corresponding eigenvector, respectively. The transformation matrix $\mathbf{W}$ can then be built using the eigenvectors of the $C$-1 non-zero eigenvalues. However, this approach has two limitations. Firstly, it requires the within-class scatter matrix $\mathbf{S}_{w}$ to be invertible, which may not necessarily be the case for time series-based DSFs. Secondly, the restriction on the number of extractable eigenvectors can adversely affect the distinguishability between classes if only a small number of classes is available for 
estimating the transformation matrix and the initial DSFs are at the same time high dimensional and with only low to moderate damage sensitivities.

The LDA-FKT, as a combination of the LDA and the FKT, does not suffer from the aforementioned limitations of the individual techniques and can be performed efficiently [53]. First, the total scatter matrix, $\mathbf{S}_{T}=\mathbf{S}_{b}+\mathbf{S}_{w}$, can be factorized using eigendecomposition as follows:

$$
\mathbf{S}_{T}=\left[\begin{array}{ll}
\mathbf{U} & \mathbf{U}_{\perp}
\end{array}\right]\left[\begin{array}{ll}
\mathbf{D} & \mathbf{0} \\
\mathbf{0} & \mathbf{0}
\end{array}\right]\left[\begin{array}{l}
\mathbf{U}^{T} \\
\mathbf{U}_{\perp}^{T}
\end{array}\right]
$$

where $\mathbf{0}$ denotes null matrices of appropriate dimensions, and matrix $\mathbf{U}$ contains the eigenvectors associated with the non-zero eigenvalues of $\mathbf{S}_{T}$ stored in the diagonal matrix $\mathbf{D}=\operatorname{diag}\left\{\lambda_{1}, \lambda_{2}, \ldots, \lambda_{r}\right\}$. The null space spanned by the eigenvectors contained in matrix $\mathbf{U}_{\perp}$ corresponding to the zero-valued eigenvalues, does not have any class-discriminatory information [68], thus it can be discarded. In the next step, a whiting transformation, $\mathbf{P}$, defined as:

$$
\mathbf{P}=\mathbf{U D}^{-1 / 2}
$$

is applied to the scatter matrices as follows:

$$
\tilde{\mathbf{S}}_{b}=\mathbf{P}^{T} \mathbf{S}_{b} \mathbf{P}, \tilde{\mathbf{S}}_{w}=\mathbf{P}^{T} \mathbf{S}_{w} \mathbf{P}
$$

The transformed matrices are related to each other as follows:

$$
\tilde{\mathbf{S}}_{b}+\widetilde{\mathbf{S}}_{w}=\mathbf{I}
$$

where $\mathbf{I}$ is an identity matrix of an appropriate dimension. Accordingly, the two matrices share the same eigenspace and can be simultaneously diagonalised [53]:

$$
\tilde{\mathbf{S}}_{b}=\mathbf{Q} \mathbf{\Lambda}_{b} \mathbf{Q}^{T}, \tilde{\mathbf{S}}_{w}=\mathbf{Q} \mathbf{\Lambda}_{w} \mathbf{Q}^{T}
$$

where $\mathbf{Q}$ is the matrix of common eigenvectors. By substituting Eq. (16) into Eq. (15) and eliminating the eigenvector matrices, the following relationship can be obtained:

$$
\Lambda_{b}+\Lambda_{w}=\mathbf{I}
$$

where the $r$-dimensional eigenvalue matrices of the whitened between-class and within-class scatter matrices are $\boldsymbol{\Lambda}_{b}=\operatorname{diag}\left\{\lambda_{b, 1}, \lambda_{b, 2}, \ldots, \lambda_{b, r}\right\}$ and $\boldsymbol{\Lambda}_{w}=\operatorname{diag}\left\{\lambda_{w, 1}, \lambda_{w, 2}, \ldots, \lambda_{w, r}\right\}$, respectively. Since the whitened scatter matrices are positive semi-definite and assuming that the 
eigenvalues of the between-class scatter matrix, $\lambda_{b, i}$, are arranged in descending order, the eigenvalues of the within-class scatter matrix, $\lambda_{w, i}$, obey the following:

$$
1 \geq \lambda_{w, 1}=1-\lambda_{b, r} \geq \lambda_{w, 2}=1-\lambda_{b, r-1} \geq \cdots \geq \lambda_{w, r}=1-\lambda_{b, 1} \geq 0
$$

The eigenvectors corresponding to the largest eigenvalues of $\widetilde{\boldsymbol{S}}_{b}$ are the most important for separating the classes, while they give the smallest intra-class variability. The combined transformation matrix, $\mathbf{T}$, defined as follows:

$$
\mathbf{T}=\mathbf{P Q}=\left[\begin{array}{llll}
\mathbf{t}_{1} & \mathbf{t}_{2} & \cdots & \mathbf{t}_{r}
\end{array}\right]
$$

can be used to transform the initial DSFs to the LDA-FKT scores, $\mathbf{v}_{i}^{F K}$, as follows:

$$
\mathbf{V}^{F K}=\mathbf{T}^{T} \mathbf{V}^{P A C}=\left[\begin{array}{llll}
\mathbf{v}_{1}^{F K} & \mathbf{v}_{2}^{F K} & \cdots & \mathbf{v}_{n_{i}}^{F K}
\end{array}\right]
$$

where $\mathbf{V}^{F K}$ is the LDA-FKT score matrix. The LDA-FKT transformation vectors, $\mathbf{t}_{i}$, are generally non-orthogonal to each other, and the resulting scores are not necessarily statistically independent. This is because for DSFs from different structural states their variance-covariance matrices can generally have different principal directions.

\subsection{Statistical hypothesis testing and damage sensitive feature ranking}

In the proposed framework, statistical hypothesis testing is used for deciding quickly if a structure is healthy or damaged. A statistical model of the z-transformed PACCs as well as the LDA-FKT scores obtained from these initial DSFs can be described by multivariate Gaussian distributions, $\mathrm{N}(\boldsymbol{\mu}, \boldsymbol{\Sigma})$, with true means, $\boldsymbol{\mu}$, and variance-covariance matrices, $\boldsymbol{\Sigma}$, respectively. Under an assumption that damage manifests itself as a shift in the mean of a DSF vector, the following statistical hypotheses can be defined:

$$
\begin{array}{lll}
H_{0}: & \boldsymbol{\mu}_{c}=\boldsymbol{\mu}_{h} & \text { (healthy) } \\
H_{1}: & \boldsymbol{\mu}_{c} \neq \boldsymbol{\mu}_{h} & \text { (damaged) }
\end{array}
$$

The null hypothesis, $H_{0}$, represents the healthy state characterised by the equality of the multivariate means from the current state, $\boldsymbol{\mu}_{c}$, and the reference healthy state, $\boldsymbol{\mu}_{h}$. The alternative hypothesis, $H_{1}$, refers to a damaged structure.

The $T^{2}(m, a, b)$ statistic is the standardized statistical distance between two estimated means, $\widehat{\boldsymbol{\mu}}_{a}$ and $\widehat{\boldsymbol{\mu}}_{b}$, of dimension $m$, and is defined as [69]:

$$
T^{2}(m, a, b)=n_{a} n_{b}\left(\hat{\boldsymbol{\mu}}_{b}-\hat{\boldsymbol{\mu}}_{a}\right)^{T} \hat{\boldsymbol{\Sigma}}_{p l}\left(\hat{\boldsymbol{\mu}}_{b}-\hat{\boldsymbol{\mu}}_{a}\right) /\left(n_{a}+n_{b}\right)
$$


where $\widehat{\boldsymbol{\Sigma}}_{p l}$ is the estimated pooled variance-covariance matrix defined as follows:

$$
\hat{\boldsymbol{\Sigma}}_{p l}=\left[\left(n_{a}-1\right) \hat{\boldsymbol{\Sigma}}_{a}+\left(n_{b}-1\right) \hat{\boldsymbol{\Sigma}}_{b}\right] /\left(n_{a}+n_{b}-2\right)
$$

The matrices $\widehat{\boldsymbol{\Sigma}}_{a}$ and $\widehat{\boldsymbol{\Sigma}}_{b}$ are unbiased estimators of the corresponding true variancecovariance matrices obtained by using $n_{a}$ or $n_{b}$ numbers of samples, respectively. The $T^{2}(m, a, b)$ statistic follows Hotelling's distribution, $T_{m, n_{a}+n_{b}-2}^{2}$, with $m$ and $n_{a}+n_{b}-2$ degrees of freedom. With the help of the $T_{m, n_{a}+n_{b}-2}^{2}$ inverse cumulative distribution function, $F_{T_{m, n_{a}+n_{b^{-2}}}^{2}}^{-1}(1-\alpha)$, the above hypotheses can be tested as follows:

$$
\begin{array}{ccc}
T^{2}(m, h, c) \leq F_{T_{m, n_{h}+n_{c}-2}^{-1}}^{-1}(1-\alpha) & \Rightarrow H_{0} \text { is accepted } \\
\text { Else } & \Rightarrow H_{0} \text { is rejected }
\end{array}
$$

where $\alpha$ is the selected level of significance, and the generic state indicators $a$ and $b$ are replaced by specific references to the healthy and current state, $h$ and $c$, respectively.

Dividing the $T^{2}(m, a, b)$ statistics by the corresponding inverse cumulative distribution function value $F_{T_{m, n_{a}+n_{b}-2}^{2}}^{-1}(1-\alpha)$ at a selected level of significance, $\alpha$, defines the following relative statistical distance:

$$
T_{r e l}^{2}(m, a, b)=T^{2}(m, a, b) / F_{T_{m, n_{a}+n_{b}-2}^{2}}^{-1}(1-\alpha)
$$

where $T_{r e l}^{2}(m, a, b) \geq 1$ indicates damage detection. Increasing the dimensionality of the DSF, $m$, by adding new components will increase the $T^{2}(m, a, b)$ distance, however, there will also be an increase in the value of the detection threshold defined by $F_{T_{m, n_{a}+n_{b}-2}^{2}}^{-1}(1-\alpha)$, because it increases with the number of statistical degree of freedom, $m$. Thus, the optimal selection of DSFs can be taken as that which maximises the relative distance $T_{r e l}^{2}(m, a, b)$.

Nevertheless, for ranking of DSFs in a multi-class setting rather than a two-class one, further modifications are necessary. For SDD, the minimum of the relative distances between the reference healthy DSFs and DSFs from $C_{D}$ distinct reference damage states is defined as the objective function for optimal DSF selection as follows:

$$
T_{S D D}^{2}(m)=\min \left\{T_{r e l}^{2}(m, h, 1), T_{r e l}^{2}(m, h, 2), \ldots, T_{r e l}^{2}\left(m, h, C_{D}\right)\right\}
$$

In this case, every distance is calculated from the healthy state data and then the minimum taken, because the goal is to ensure all the damage states are separated as much as possible 
from the healthy state. This can be achieved by finding a DSF selection of size $m$ that maximises $T_{S D D}^{2}(m)$.

For maximising the separability of multiple classes for SDL, the following extension of $T_{r e l}^{2}(m, a, b)$ is proposed to select the optimal DSF subset:

$$
T_{S D L}^{2}(m)=\sum_{\substack{a=1 \\ a \neq h}}^{C_{D}-1} \sum_{\substack{b=a+1 \\ b \neq h}}^{C_{D}} T_{r e l}^{2}(m, a, b)
$$

Note that one class (i.e. the healthy state) is excluded from calculating $T_{S D L}^{2}(m)$ as we assume SDD will have been conducted earlier.

In the FF ranking process [69], these variables that improve an objective function are consecutively selected to compose an optimal subset of DSFs for a statistical analysis. This method is characterized by its moderate computational requirements, ease of implementation and short processing times. Therefore, it is herein applied for ranking the univariate DSFs with respect to either $T_{S D D}^{2}(m)$ or $T_{S D L}^{2}(m)$ as the objective functions for SDD or SDL, respectively. In the first FF iteration, all the individual components are evaluated against the objective function and the one with the highest value is selected. It is the most sensitive DSF component and receives the highest rank. Then, all the remaining components are added in turn to the first selected component and the one corresponding to the largest bivariate distance is retained with rank two. The procedure continues until all the components have been included in the DSF vector. The optimal selection and size of the DSF vector is the one that maximises the objective function. It should be noted that the FF ranking only guarantees finding the global maximum if all DSFs are statistically independent, i.e. the variancecovariance matrix is diagonal. However, for small to moderate correlations amongst the DSF the outcome is typically not strongly affected and computational efficiency gains outweigh the risk of arriving at a suboptimal solution.

\subsection{Hierarchical neuro-fuzzy modelling}

The characteristics of DSFs generally depend not only on the existence of damage but also on its location and extent. Thus, the use of an advanced classification method is required to avoid misclassifications caused by damage scenarios that are potentially different and not used in the training process. The ANFISes are Takagi-Sugeno fuzzy inference systems with theoretical universal approximation power [55], which enables them to represent any non- 
linear function arbitrarily well. Thus, they can be used to perform SDL by non-linearly mapping DSFs, as inputs, to damage locations, as outputs, even if distinct DSF characteristics are related to the same location because of, e.g., varying damage severities. Nevertheless, for high dimensional DSFs there are many inputs, and creating and training of an ANFIS can become computationally prohibitive due to the so called 'curse of dimensionality' experienced by soft computing networks. It can also be expected that including features that are noisy or only weakly altered by damage (i.e. its presence and location in this case) will adversely affect the accuracy of predictions as well as the model complexity.

To address these challenges, a system with a hierarchical architecture comprising several ANFISes and referred to as HANFIS is developed as illustrated in Fig. 2. Each square represents a single ANFIS with two inputs and one output (explained itself later in Fig. 3). The HANFIS inputs are the ranked univariate DSFs, $v_{i}$, where index $i$ refers here to the rank. (Both previously introduced DSF types, i.e. the PACF z-scores and the LDA-FKT scores, are studied, thus the generic notation $v_{i}$ is used by neglecting the superscript.) By using DSF components as inputs in the order they are ranked, their variable sensitivities to damage can dictate the model structure, i.e. the number of ANFISes to be used. The first hierarchical level ANFIS uses the two most sensitive DSFs as inputs. It is trained against the desired outputs, i.e. the correct damage locations. It is assumed that this is the best possible model with two inputs, but it may not necessarily be the overall best model because including more DSF components can improve the SDL accuracy if they are sufficiently sensitive to damage locations. However, simply increasing the number of inputs into a single ANFIS quickly becomes computationally prohibitive. Therefore, the proposed hierarchical structure grows by adding another ANFIS that uses as inputs one new ranked DSF component and the predicted output of the previous HANFIS. In this way, the predicted outputs from the lower hierarchical level ANFISes are propagated until an optimal hierarchical level is reached. The corresponding HANFIS uses inputs up to the optimal ranked univariate DSF, $v_{\text {opt }}$. Identifying this optimal hierarchical level may require using many, possibly all the available inputs up to the $m$-th DSF component, $v_{m}$, but since training of each ANFIS is done separately the overall computational effort increases only linearly with the number of hierarchical levels. This desirable behaviour extends easily the applicability of the proposed model structure to high dimensional DSFs. The root mean square (RMS) error of the $h$-th ANFIS, $\varepsilon_{h}$, can be calculated for $n$ samples of the target outputs, $y_{i}$, and the predicted outputs, $\hat{y}_{h, i}$, as: 


$$
\varepsilon_{h}=\sqrt{\sum_{i=1}^{n}\left(y_{i}-\hat{y}_{h, i}\right)^{2}}
$$

Evaluating this error for HANFISes with different hierarchical levels enables identifying the optimal level, opt. However, using only a single dataset for HANFIS architecture optimisation may easily lead to overfitting. Furthermore, overly large models might be produced. Insights into the approximation and estimation errors obtained from $k$-fold crossvalidation can be used for more robust model identification [70]. For this type of crossvalidation, a dataset is divided into $k$ subsets of randomly selected samples without replacement. Then, $k$ models are trained using only $k-1$ sample subsets. The retained unseen samples enable to calculate the validation errors of the separate models. Therefore, a model selection criterion, $\eta_{h}$, is defined for this task as:

$$
\eta_{h}=\hat{\mu}_{\varepsilon_{h}}+\hat{\sigma}_{\varepsilon_{h}}
$$

where $\hat{\mu}_{\varepsilon_{h}}$ and $\hat{\sigma}_{\varepsilon_{h}}$ are the estimates of the mean and standard deviation of the error from the cross-validation estimates. The minimum of this expression is selected as the optimal hierarchical level. Then, following the recommended practice [66], the final modelling of the identified HANFIS is performed using all available data.

The individual ANFISes have the same structure, which is shown for the $h$-th ANFIS in Fig. 3 as an example. Here, square blocks represent nodes with adaptive parameters, while circles indicate fixed nodes without adjustable parameters. It resembles an ANN with two inputs, one output and five layers. In the first layer, the membership grades are evaluated for the two inputs using $q$ membership functions, $M_{1, i}^{h}$ and $M_{2, i}^{h}(i=1,2, \ldots, q)$, respectively. They typically are non-linear functions, such as Gaussian or sigmoid functions, and their parameters are the so-called premise parameters of the fuzzy inference system. The membership functions are adaptive nodes because their parameters are modified during training. The nodes in the second layer, denoted by $\Pi_{i}$, are fixed and calculate firing strengths, $w_{i}^{h}$, by multiplying the incoming signals with each other. The firing strengths are normalized by dividing them by $\sum_{i=1}^{q} w_{i}^{q}$ to $\bar{w}_{i}^{h}$ in the next layer nodes denoted as $N_{i}$. The forth layer, whose nodes are denoted as $F_{i}^{h}$, evaluates the $q$ fuzzy if-then rules given as:

$$
\text { Rule } i \text { : if } y_{h-1} \text { is } M_{1, i}^{h} \text { and } v_{h+1} \text { is } M_{2, i}^{h} \text {, then } f_{i}^{h}=p_{i}^{h} y_{h-1}+q_{i}^{h} v_{h+1}+r_{i}^{h}
$$


where $p_{i}^{h}, q_{i}^{h}$ and $r_{i}^{h}$ the so-called consequent parameters describing linear output membership functions. The corresponding nodes are thus adaptive. Finally, the overall output, $y_{h}$, is computed in a single fixed node $\Sigma$ in layer five as a weighted sum of all the incoming signals. The ANFIS structure allows using the well-established ANN training procedures for optimizing its parameters. A hybrid learning strategy is applied in this research, which combines the forward and back-propagation learning [54]. In the forward pass, the linear consequent parameters are estimated with the help of a least-squares procedure. Then, the error signals are back-propagated to the membership functions, where a gradient-descent approach is applied for optimizing the non-linear premise parameters. The error for a given training dataset is iteratively reduced by performing several epochs of forward and backward passes. To avoid overfitting of the individual ANFISes, a hold-out cross-validation is applied, where a separate checking dataset is used for calculating the checking error. The epoch with the minimum checking error gives the optimal parameters.

The number of membership functions and rules, $q$, depends on the properties of the inputs. Due to the close relationship of this type of fuzzy inference systems with cluster analysis [55], unsupervised subtractive clustering can be used for determining an appropriate ANFIS structure for each hierarchical level. Although other clustering techniques can be employed, e.g. k-means, fuzzy c-means or Gaussian mixture models, subtractive clustering is simple and efficient. Cluster centres are identified iteratively using a density measure estimated for each input sample, which is modified after each iteration by removing the effect of the identified clusters.

\section{BIVARIATE PROBLEM ILLUSTRATION}

This section discusses the selection of the original and LDA-FKT transformed DSFs for enhanced SDD and SDL. The discussion employs a generic $m$-variate original DSF, $\mathbf{v}$, which is assumed to follow in a certain structural state, $s t$, a Gaussian distribution, $\mathrm{N}\left(\boldsymbol{\mu}_{s t}, \boldsymbol{\Sigma}_{s t}\right)$, with the true mean vector, $\boldsymbol{\mu}_{s t}$, and variance-covariance matrix, $\boldsymbol{\Sigma}_{s t}$, respectively. The LDA-FKT version of the feature is denoted by $\mathbf{v}^{F K}$. For the sake of simplicity but without loss of generality, it is assumed that the statistical uncertainty of the DSF true mean vector and the variance-covariance matrix estimates for the healthy state, $s t=h$, are negligible. Thus, statistical hypothesis testing for single DSF vector samples in the current state, $\mathbf{v}_{\text {curr }}$, can be done with the help of the squared Mahalanobis distance, $D^{2}(m)$, defined as [69]:

$$
D^{2}(m)=\left(\mathbf{v}_{\text {curr }}-\boldsymbol{\mu}_{h}\right)^{T} \boldsymbol{\Sigma}_{h}^{-1}\left(\mathbf{v}_{\text {curr }}-\boldsymbol{\mu}_{h}\right) \square \mathrm{X}_{m}^{2}
$$


which permits using a simpler $\Xi_{m}^{2}$ distribution with $m$ DOFs rather than the more general Hotelling's distribution, $T_{m, n_{a}+n_{b}-2}^{2}$. The $\Xi_{m}^{2}$ inverse cumulative distribution function, $F_{\Xi_{m}^{2}}^{-1}(1-\alpha)$, can be used to define a statistical threshold at a selected level of significance, $\alpha$.

To be able to visualize the problem, a bivariate DSF vector is considered in the following discussions, i.e. $m=2$ from now on. The difference between the current state DSF vector, $\mathbf{v}_{c u r r}$, and the true mean in the healthy state, $\boldsymbol{\mu}_{h}$, is given as:

$$
\Delta \mathbf{v}=\left[\begin{array}{c}
v_{\text {curr }, 1}-\mu_{h, 1} \\
v_{\text {curr }, 2}-\mu_{h, 2}
\end{array}\right]=\left[\begin{array}{l}
\Delta v_{1} \\
\Delta v_{2}
\end{array}\right]
$$

For simplicity, it is further assumed that the standard deviations of both initial DSF components are equal to one. Thus, the variance covariance matrix in the healthy state is:

$$
\boldsymbol{\Sigma}_{h}=\left[\begin{array}{cc}
1 & \rho_{12} \\
\rho_{12} & 1
\end{array}\right]
$$

where $\rho_{12}$ is the correlation coefficient. Accordingly, the statistical test using the squared Mahalanobis distance $D^{2}(m)$ reduces to:

$$
D^{2}(2)=\frac{\Delta v_{1}^{2}+\Delta v_{2}^{2}-2 \rho_{12} \Delta v_{1} \Delta v_{2}}{1-\rho_{12}^{2}} \leq F_{\mathrm{X}_{2}^{2}}^{-1}(1-\alpha)
$$

and uses the bivariate distribution $\Xi_{2}^{2}$.

In what follows, two cases are discussed to highlight that, depending on the problem at hand, either the original or transformed feature selections may be optimal for SDD and SDL.

\subsection{Case 1}

Case 1 was conceived to demonstrate that using the LDA-FKT scores may be optimal for both SDD and SDL, albeit the best scores that should be selected for each task may be different.

In addition to the healthy state, three hypothetical damage states, namely $\mathrm{A}, \mathrm{B}$ and $\mathrm{C}$, are considered. The DSF true mean vectors for these damage states are assumed as follows:

$$
\boldsymbol{\mu}_{\mathrm{A}}=\left[\begin{array}{l}
3.8-\mu_{h, 1} \\
3.8-\mu_{h, 2}
\end{array}\right], \boldsymbol{\mu}_{\mathrm{B}}=\left[\begin{array}{c}
-5.3-\mu_{h, 1} \\
-2.9-\mu_{h, 2}
\end{array}\right], \boldsymbol{\mu}_{\mathrm{C}}=\left[\begin{array}{c}
0.7-\mu_{h, 1} \\
-6.1-\mu_{h, 2}
\end{array}\right]
$$


Equation (34) allows defining an elliptical acceptance region of the null hypothesis (Eq. (21) ). This is shown in Fig. 4a for a selected illustrative correlation coefficient $\rho_{12}=0.3$ and significance level $\alpha=5 \%$. To make the discussion more tractable, it is assumed that the DSF variance-covariance matrices in the damage states are identical to the healthy state one shown in Eq. (33). Their DSF distributions have therefore the same shape, but occur in different locations as shown in Fig. 4a. It should be noted that circular DSF distributions are a special case for uncorrelated initial DSFs, i.e. when $\rho_{12}=0$.

In addition to the elliptical acceptance region of the null hypothesis applicable when using bivariate DSFs, acceptance regions are constructed for the case when only either original DSF $v_{1}$ or DSF $v_{2}$ is used. They appear as vertical and horizontal bands bounded by $\pm \sqrt{F_{\Xi_{1}^{2}}^{-1}(1-\alpha)}$ considering the univariate distribution $\Xi_{1}^{2}$.

For the LDA-FKT scores, $v_{1}^{F K}$ and $v_{2}^{F K}$, to be used for SDD, the true mean vectors of all four classes should be used to compute the between-class scatter matrix, $\mathbf{S}_{b}$, using Eq. (8), and the within-class scatter matrix, $\mathbf{S}_{w}$, using Eq. (10). To further simplify the problem, we assume that the numbers of samples available from each of the four structural states introduced thus far are the same and large. The LDA-FKT transformation matrix, T, (Eq. (19) ) is obtained as:

$$
\mathbf{T}=\left[\begin{array}{ll}
\mathbf{t}_{1} & \mathbf{t}_{2}
\end{array}\right]=\left[\begin{array}{cc}
-0.82 & 0.46 \\
0.58 & 0.89
\end{array}\right]
$$

where the matrix columns (i.e. transformation vectors, $\mathbf{t}_{1}$ and $\mathbf{t}_{2}$ ) are normalized for convenience to unit length. It can easily be verified that they are not orthogonal. The coordinate system axes defined by the two vectors are also shown in Fig. 4a. The initial DSFs can then be transformed to their LDA-FKT scores, $v_{1}^{F K}$ and $v_{2}^{F K}$, with the help of Eq. (20). Furthermore, acceptance bands are constructed for $v_{1}^{F K}$ and $v_{2}^{F K}$ using the statistical threshold $\pm \sqrt{F_{\Xi_{1}^{2}}^{-1}(1-\alpha)}$. These bands appear as inclined (and perpendicular to the respective transformation vectors) and their widths are not identical, i.e. the acceptance band for $v_{1}^{F K}$ is wider than that for $v_{2}^{F K}$.

Examining Fig. 4a, for statistical hypothesis testing using selected univariate DSFs for $\mathrm{SDD}$, where we only want to be able to check if the current data is statistically different from 
those of the healthy state but are not concerned about which particular damage state they come from, the following observations can be made. If the LDA-FKT scores $v_{2}^{F K}$ are used, then data coming from any of the three damage states A, B and C can easily be separated from the healthy state. Only a small number of false positive damage detection errors is expected and even a smaller number of false negative errors. In contrast, projecting the initial DSFs to form $v_{1}^{F K}$ allows for easy detection of data from damage state $\mathrm{C}$ as different from the healthy state, while data from damage state B can only be partially differentiated from the healthy state, and data from damage state A will mostly be undistinguishable from the healthy state. For SDD based on selected initial DSF components, it can be observed that using $v_{1}$ offers a similarly confused performance to using $v_{1}^{F K}$, only this time data from damage state B can easily be separated from the healthy state, data from damage state A only partially, and data from damage state $\mathrm{C}$ is mostly undistinguishable. Using $v_{2}$ only, differentiation of data from damage state $\mathrm{C}$ from the healthy state data is clear, but data from damage states A and B can only be partially correctly identified as coming from a damaged structure.

Note that for bivariate hypothesis testing the same ellipse defines the acceptance region for both the original and the transformed features. Using bivariate hypothesis testing for SDD, i.e. considering jointly both original features or both their LDA-FKT projections enables easy separation of damage states $\mathrm{B}$ and $\mathrm{C}$ from the healthy one, but the ellipse for damage state A shows a degree of overlap with the healthy state one. This overlap will give rise to a modest number of false positive or false negative damage detection errors. Whether the performance of the proposed SDD algorithm with $v_{2}^{F K}$ or a bivariate DSF can be considered better will depend on the user's tolerance for two types of errors. Overall, however, for this SDD example the LDA-FKT score $v_{2}^{F K}$ outperformed all other univariate DSFs and was shown to be competitive against both bivariate DSFs, demonstrating that in this case it can be optimal to both transform DSFs and retain only a selected transformed feature.

In the SDL problem, having determined that the structure is damaged, we now ignore the healthy state and focus on detecting which specific damage state occurred. The figure to discuss this situation is Fig. 4b. Here the LDA-FKT transformation matrix, T, (Eq. (19)) obtained using only the DSF distributions of the three damage states is 


$$
\mathbf{T}=\left[\begin{array}{ll}
\mathbf{t}_{1} & \mathbf{t}_{2}
\end{array}\right]=\left[\begin{array}{cc}
-0.80 & 0.51 \\
0.60 & 0.86
\end{array}\right]
$$

Differentiating successfully between the various considered damage states requires that their statistical distances, measured along the axis in question for univariate features or from the origin of the coordinate system for bivariate ones, be sufficiently different. Figure $4 \mathrm{~b}$ demonstrates that this can be most successfully done using the LDA-FKT score $v_{1}^{F K}$ : the statistical distance measured along this axis is the largest for damage state $\mathrm{C}$, followed by damage state B and then damage state A. It can be seen that there is a degree of overlap between the three damage state DSF distributions in that direction, and so SDL will not be without misclassifications. This, however, is the property of the assumed data, and other datasets with more distant means and/or less scatter would lead to improved SDL outcomes. To contrast this optimal, albeit imperfect, performance with less successful choices of DSF, we can see that using $v_{2}^{F K}$ only results in rather similar distances for all three damage states; using either $v_{1}$ or $v_{2}$ only will give insufficiently different distances between damage states $\mathrm{A}$ and B; and bivariate DSFs cannot separate damage state C from B. The simple SDL example shows again that the winning strategy is to both transform and retain only a selected transformed feature, but this feature may be different than the previous one chosen for SDD.

For application of HANFISes for SDL, the use of initial DSFs would require four Gaussian membership functions in the input layer, i.e. two for each DSF component. Additionally, two rules and consecutively two linear output membership functions would be needed to distinguish all three damage states. An LDA-FKT HANFIS requires only three Gaussian membership functions in the input layer, one rule and one linear output membership function. This fact extends the benefits of LDA-FKT for SDL for the use with neuro-fuzzy models, where parsimonious model structures with small numbers of inputs are desirable for limiting computational efforts.

\subsection{Case 2}

Case 2 was selected in contrast to Case 1 to highlight there may be situations where using the LDA-FKT scores may be suboptimal for at least one of SDD and SDL and the original features may be preferred. The case is illustrated in Figure 5a and b for SDD and SDL, respectively.

Here, the assumed DSF true mean vectors for damage states A, B and C are as follows: 


$$
\boldsymbol{\mu}_{\mathrm{A}}=\left[\begin{array}{c}
4.6-\mu_{h, 1} \\
0.6-\mu_{h, 2}
\end{array}\right], \boldsymbol{\mu}_{\mathrm{B}}=\left[\begin{array}{c}
4.6-\mu_{h, 1} \\
6.0-\mu_{h, 2}
\end{array}\right], \boldsymbol{\mu}_{\mathrm{C}}=\left[\begin{array}{c}
-4.7-\mu_{h, 1} \\
5.7-\mu_{h, 2}
\end{array}\right]
$$

Performing a similar analysis for SDD as in Case 1 reveals that for Case 2 the optimal DSF is the original feature $v_{1}$, i.e. it does not pay off to transform features but their judicious selection is still desirable. For SDL, on the other hand it is $v_{1}^{F K}$ that outperforms all remaining univariate or bivariate options. Case 2 further confirms that DSF transformation and selection need to be tailored to the specific task and patterns present in the data.

It should be born in mind that the presented two cases are simple, illustrative and represent only particular situations. Real applications will generally be more challenging because DSF vectors will have significantly higher dimensions, the structural states may be far less clearly separated and the variance-covariance structures may change with damage. Nevertheless, the two cases illustrate the potential benefits of selecting carefully from amongst the initial and LDA-FKT modified DSFs for enhanced SDD and SDL performance. They also clearly demonstrate the need for a systematic assessment of DSFs for specific SHM tasks, as proposed and later examined in this study on experimental data using the FF method in combination with specialised objective functions.

\section{EXPERIMENTS}

Physical experiments are essential for demonstrating and verifying the proposed concepts. Therefore, laboratory experiments with a small WTB were performed. This section describes first the specimen and the experimental configuration. Then, the estimation of the PACF from vibrational responses is presented in preparation for hypothesis testing and LDA-FKT neurofuzzy modelling and the evaluation of the SDD/SDL algorithms.

\subsection{Experimental configuration}

The specimen for the dynamic experiments is a $2.36 \mathrm{~m}$ long WTB of a residential-size WT with $5 \mathrm{~m}$ rotor diameter and a rated power output of $5 \mathrm{~kW}$. Its cross-section has a constant width of $150 \mathrm{~mm}$ and is defined by the aerofoil profile E387 with a curved low-pressure side and flat high-pressure side, as shown in Fig. 6. The solid WTB section is made by pultrusion of a glass-fibre reinforced epoxy composite. The total mass was measured as 7,111 $\mathrm{g}$ and the mass density estimated as $2.30 \mathrm{~g} / \mathrm{cm}^{3}$. The WTB was set up vertically for the experiments to save laboratory space, with the root clamped to a massive steel base sitting on a thick concrete floor (see Fig. 7). 
In a preliminary study, the WTB's dynamic characteristics were identified by experimental modal analysis from accelerance frequency response functions from experiments with an instrumented hammer. The high flexibility of the WTB is demonstrated by the low first natural frequency of $1.75 \mathrm{~Hz}$. This is an important property for real applications, where large, highly flexible WTBs can be excited by low frequency wind pressure fluctuations. Wind-like excitation is simulated in this study using a household pedestal fan with a rotor diameter of $40.6 \mathrm{~cm}$ and a height of $62 \mathrm{~cm}$ measured from the WTB root. The fan was positioned at about $1 \mathrm{~m}$ from the WTB leading edge with an orientation corresponding to a zero-degree angle of attack. Its operation power can be selected in three levels up to a maximum of $40 \mathrm{~W}$, where the second level is used in this study. The use of a fan enables to apply continuous, non-contact excitation. This type of experimental setup allows simulating a realistic scenario of a parked WT with a feathered WTB in upright position under wind-like excitation.

For demonstrating the performance of SDD and SDL algorithms without permanently altering the specimen, changes were introduced non-destructively by attaching small masses between $10 \mathrm{~g}$ and $100 \mathrm{~g}$ in increments of $10 \mathrm{~g}$ at selected locations on the WTB. These locations were chosen based on inspection reports and WTB damage studies [71-74]. Three damage hotspots were indicated: the WTB tip (T), the leading edge (LE) at $66 \%$ of the blade length from the root, and the trailing edge (TE) at $33 \%$ of the blade length from the root. For supervised global SDL methods, a pragmatic approach is to focus on potential damage locations with high prevalence rates and the risk they pose to structural integrity. This allows reducing efforts and costs for training of algorithms, while adequate levels of safety and reliability can be assured. Nevertheless, to assess the generalization capabilities of the proposed methods, the effect of variations in the extent and location of damages was investigated by means of additional damage scenarios. A summary of the studied damage states used for training and testing is given in Table 1, while locations are indicated in Fig. 7. The additional scenarios are indicated in Table 1 as TE/LE $\pm 5 \mathrm{~cm}$ and refer to locations $5 \mathrm{~cm}$ higher or lower, respectively, than the main TE and LE locations.

Response accelerations were measured using four miniature piezoelectric accelerometers model Metra KS94B-100 with sensitivities of approximately $100 \mathrm{mV} / \mathrm{g}$ and frequency ranges of $0.5 \mathrm{~Hz}$ to $28 \mathrm{kHz}$. They were attached with adhesive wax to the highpressure side of the WTB at the locations marked as S1-4 in Fig. 7. Accelerations were digitized with a National Instruments NI-9234 data acquisition card connected to a National 
Instruments cDAQ-9174 chassis. Signals were processed with the National Instruments software LabView installed on a laptop computer.

\subsection{Partial autocorrelation function identification}

Acceleration responses were acquired for 30 min with a constant sampling rate of 2,048 Hz. A typical response of the healthy WTB at sensor S1 is shown in Fig. 8, where fluctuations generally slightly exceed $0.05 \mathrm{~g}$. To reduce the amount of high-frequency measurement noise, data pre-processing started with low-pass filtering using an eighth order Chebyshev type I filter with a cut-off frequency of $409.6 \mathrm{~Hz}$ and resampling at $512 \mathrm{~Hz}$.

For creating a database of DSFs, each signal was divided into 400 segments of $5 \mathrm{~s}$ length with an overlap of approximately $10 \%$. These segments were normalised by removing their estimated means and dividing by their estimated standard deviations to remove possible variations caused by fluctuations of the excitation. Then, PACFs were estimated from these time series segments. Statistical testing of the PACC estimates for increasing lags allows selecting significant PACCs for the definition of a multivariate DSF [64]. This was done for the data from a healthy state measurement for all four sensors S1-4 and PACCs with lags from one to 60 were identified as significant at the $95 \%$ confidence level.

\section{STRUCTURAL DAMAGE DETECTION VIA STATISTICAL HYPOTHESIS TESTING}

This section explains first the transformation of the initial DSFs. Secondly, the optimal DSF subsets for SDD are selected with the help of the FF procedure and are then used for SDD in previously unseen data coming from damage scenarios that were either used or not used in training. This is not only applied to DSFs from separate sensors but also for a combination of DSFs from all the sensors to investigate potential benefits of fusing DSFs from several sensors and processing them jointly.

\subsection{Feature transformation and optimal subset selection for SDD}

The original DSFs (PACCs) from the healthy and six damage datasets, listed in Table 1 as training datasets (two extents of either $20 \mathrm{~g}$ or $50 \mathrm{~g}$ at one of the three locations TE, LE or T, respectively), were transformed with the help of the LDA-FKT established using 400 samples of each class. From the between-class and within-class scatter matrices of these DSFs, the whiting transformation matrix, $\mathbf{P}$, (Eq. (13)) was estimated. The eigendecomposition of the combined variance-covariance matrices (Eq. (11)) revealed that most of the eigenvalues are 
close to zero. As discussed in Section 2.2, the common null space of these matrices does not contain discriminatory information and can be discarded. Therefore, only the twelve largest eigenvalues and eigenvectors were used to compose $\mathbf{P}$ for all the sets of initial features. The common eigenvector matrices, $\mathbf{Q}$, (Eq. (16)) were then calculated by eigendecomposition of the whitened scatter matrices, where using the within-class scatter matrices lead to more stable results than the between-class scatter matrices because their ranks are not limited to the number of classes. By combining $\mathbf{P}$ and $\mathbf{Q}$ matrices, the actual LDA-FKT matrix, $\mathbf{T}$, (Eq. (19) ) was obtained and used for transforming the initial DSFs to scores, $\mathbf{v}^{F K}$, (Eq. (20)).

In the next step, the FF procedure was applied to both the LDA-FKT scores and to the PACC-based initial DSFs for performance comparison of the two feature types using the same datasets. For SDD, the FF algorithm used $T_{S D D}^{2}(m)$ (Eq. (26)) as the objective function at $5 \%$ significance. The results for both DSF types are shown in Fig. 9. Beginning the discussion with the initial DSFs, it can be seen that using jointly the PACCs estimated from all the sensors outperformed the DSFs of individual sensors with the optimal performance achieved for 39 ranked PACCs and $T_{S D D}^{2}(m)$ of 137.50. For separate sensors, the best results for sensors S1-4 were respectively 34.59, 33.55, 42.35 and 60.67. Comparing these observations sensor-by-sensor and for all the sensors with the results for the LDA-FKT scores shows that the initial DSFs performed better in all the cases. However, sensor S3 gave a $T_{S D D}^{2}(m)$ of 41.64 , the overall highest objective function for the LDA-FKT scores, whereas sensors S1, S2 and S4 yielded 29.29, 20.39 and 28.37, respectively. Furthermore, using all the sensors led to the worst performance with a relative distance of only 19.74. Nevertheless, in all the cases considered the relative distances were larger than one, thus every setting enabled detection of all the studied damage scenarios.

\subsection{Statistical structural damage detection}

Using the optimally selected DSF subsets, SDD was performed, with the training healthy dataset reused for estimating the reference DSF statistic. Then, single samples from previously unseen healthy and damage state data of 400 samples for each class were used for calculating the $T^{2}(m, a, b)$ statistic (Eq. (22)). The performance for binary SDD classification (i.e. healthy or damaged) can be evaluated by means of the receiver operating characteristic (ROC) curves. In these curves, true positive rates from correctly classified damage states are plotted against false positive rates resulting from misclassified healthy state data for varying statistical thresholds instead of a single threshold [75]. An example of a ROC curve is shown 
in Fig. 10 for the selected PACCs and LDA-FKT scores obtained from all the sensors. A perfect classifier would have a bilinear ROC starting at a true positive rate and false positive rate of one for very small detection thresholds, going through true positive rate of one and false positive rate of zero, and terminating at a true positive rate and false positive rate of zero for large detection thresholds. This ideal performance can be visualized by considering classifications obtained with detection thresholds sweeping between zero and a large value for two well-separated clusters with very small inter-cluster spreads and corresponding to two different states. On the other hand, the ROC curve will be a straight diagonal line for a random classifier with $50 \%$ chance of obtaining a correct result.

The area under the receiver operating characteristic curve (AUC) can be used as a summary scalar measure of the classifier performance, where $\mathrm{AUC}=1.0$ and $\mathrm{AUC}=0.5$ correspond to a perfect and a random classifier, respectively. All the AUC values obtained are listed in Table 2 separately for the six damage scenarios previously used and 26 not used in training, where 400 samples were used for each scenario. For both cases the AUC values are identical (to two significant digits), suggesting that the proposed supervised training procedures can be applied with a comparable effect to damage scenarios not used in training. Furthermore, the classifier performance appears to depend on the sensor location, where sensor S2 yielded for both DSF types the worst results. For the selected PACCs, S4 was the best performing single sensor (AUC=0.91), followed by S1 and S3. However, using the PACCs selected from all the sensors gave the overall best result of an almost perfect classification ( $\mathrm{AUC}=0.95$ ). Using the selected LDA-FKT scores gave AUC $=0.86$ for sensors S3, S4 and the combined all sensor data as the best value for this DSF type. As can be seen, PACCs outperformed the LDA-FKT scores as DSFs for SDD when S1, S4 and all the sensors were used, whereas the LDA-FKT scores gave higher results for sensors S2 and S3. By comparing the results for separate and combined sensors, it can be seen that the latter approach provided better performance.

In addition to the AUCs, Fig. 11 presents all the individual detection results for the selected PACCs and LDA-FKT scores obtained from all the sensors. The results are presented in terms of the relative statistical distance, $T_{\text {rel }}^{2}$, (Eq. (25)) because it allows comparing DSF vectors with different dimensionalities. For this distance, the detection threshold equal unity, thus values below the threshold indicate the healthy state and values above the threshold a damaged state, respectively. It can be seen that for the healthy state measurements the distance of both DSF types generally fall below the detection threshold, which indicates a small false alarm rate. For the different damage states, a strong dependency 
between the damage locations and extents and the values of the relative distance can be observed for both DSF types (note logarithmic scale used). Thus, damages at the trailing edge and the tip have higher damage detectabilities than the leading-edge damages. Furthermore, light damages, i.e. simulated by attaching $10 \mathrm{~g}$ and $20 \mathrm{~g}$ masses, are difficult to detect, while strong damages can be detected with perfect accuracy. However, differences can also be observed between the two DSFs. The selected PACCs lead to higher relative statistic values then the selected LDA-FKT scores. Furthermore, the former allow detecting small damages with higher accuracy, e.g. the $30 \mathrm{~g}$ to $60 \mathrm{~g}$ leading edge damages. This explains the obtained higher AUC values for PACCs from all the sensors in comparison to the LDA-FKT scores from all the sensors.

The better performance when using the selected PACCs rather than the LDA-FKT scores can be explained by the fact that the LDA-FKT seeks to maximize the discriminability between DSF samples from different classes projected orthogonally onto a few directions. All the classes are treated as equally important. The obtained directions of projection are most strongly determined by the maximum differences in the DSF vector means in the betweenclass scatter matrix. Thus, the largest distances between the classes of different damage types can dominate the resulting transformation, while the distances to the healthy state DSFs might have a smaller chance to influence the transformation. Furthermore, the number of selectable components is significantly reduced. On the other hand, the selection of initial DSFs does not suffer in the same way, thus the SDD results are better for the selected PACCs. Note that which of the two, PACS or LDA-FKT scores, will perform better can only be evaluated via an actual application of the method to the data at hand.

\section{STRUCTURAL DAMAGE LOCALISATION VIA NEURO-FUZZY CLASSIFICATION}

After structural damage has been detected, the second stage of the proposed methodology is concerned with damage localisation. Separating these two tasks enables to develop and train specialised algorithms in the hope of improved performance, and a neuro-fuzzy approach is explored for SDL in this study. It is again based on the premise that including only those DSF components that can best differentiate between damage states will deliver a computationally efficient and accurate solution. This section presents first the transformation and ranking of DSFs for SDL. Then, the proposed hierarchical neuro-fuzzy modelling scheme is demonstrated. Finally, SDL results for previously unseen datasets from damage scenarios both used and not used in training are discussed. 


\subsection{Feature transformation and ranking for SDL}

In contrast to SDD, only DSFs from the six different damage states are used for estimating the SDL LDA-FKT matrices using 400 samples of each state (i.e. the healthy state data are excluded). This is because in our two-stage methodology, damage existence would have been declared by the previously described SDD algorithm before commencing SDL. As previously, by discarding the common null space of the combined scatter matrices, the ten largest eigenvalues and corresponding eigenvectors were selected for calculating the whiting transformation matrices. Then, the actual LDA-FKT matrices were computed for the DSFs from separate sensors and their fusion.

The capabilities of univariate DSFs to distinguish between the different damage states are not the same. Therefore, an FF ranking was performed with the aim to maximize the objective function $T_{S D L}^{2}(m)$ (Eq. (27)) for increasing numbers of individual DSFs. The results for the initial and LDA-FKT-transformed DSFs are shown in Fig. 12. For both DSF types, it can be observed that the DSFs from separate sensors show a similar behaviour with the highest $T_{S D L}^{2}(m)$ values obtained for low feature dimensions. Nonetheless, using DSFs from all the sensors together outperformed individual sensors for both PACCs and LDA-FKT scores, where the latter gave the overall highest objective function values. The development of HANFIS for SDL discussed in the subsequent section will take advantage of the DSF component ranking.

\subsection{Hierarchical neuro-fuzzy modelling}

The proposed hierarchical neuro-fuzzy superstructure can take advantage of the previously established DSF component ranking. To identify the optimal hierarchical levels and select the corresponding optimal DSF subsets, neuro-fuzzy models of increasing hierarchical levels were trained for predicting damage locations, as distances from the blade root, using the available damage state training datasets. All models used Gaussian membership functions. Their numbers were identified in a data-driven manner with the help of subtractive clustering, as discussed in Section 2.4. A 5-fold cross-validation was undertaken where the separate individual ANFIS trainings used a hold-out cross-validation for identifying the optimal epoch numbers. Figure 13 shows the values of the model selection criterion $\eta_{h}$ (Eq. (29)). For the PACC-based models, after a significant decrease the results stayed constant or increased again for higher hierarchical levels. Instances of probable convergence to local minima can also be seen as outlying values of the model selection criterion. The chosen optimal 
hierarchical levels are summarized in Table 3. The PACC HANFIS for sensor S2 required the lowest number of hierarchical levels but still gave results as good as sensor S3 or all the sensors. The worst performance was given by sensor S1 followed by sensor S4. Contrary, the modal selection criteria for the LDA-FKT HANFISes change with increasing orders only slightly with an exception of sensor S3, where after a small decrease the objective function increases significantly (Fig. 11). The models for sensor S2 and all the sensors gave the lowest selection criterion values, which were also the overall best. The models for sensor S1 and S4 yielded the highest selection criterion values, but they were at the same time still as good as the best results of the initial DSFs. It can be seen from Table 3 that the LDA-FKT-based models required markedly much less hierarchical levels and parameters than the PACC-based ones, thus they are attractive because of lower complexity and less computational effort. After identification of the appropriate hierarchical levels, the final modelling of all the HANFISes was performed using all the available DSF samples.

\subsection{Neuro-fuzzy structural damage localisation}

Four hundred DSFs were extracted from previously unseen datasets of each of the six damage scenarios used and 26 scenarios not used in training and presented to the identified HANFISes for validation of their SDL performance. The results for the different DSF types and sensors are summarized in Table 4 in terms of RMS errors. Comparing the PACC-based models for the damage scenarios used in training shows that utilising jointly DSFs from all the sensors gave the lowest errors, followed by sensor S3 and S2. Higher errors were obtained for sensors S1 and S4. Nevertheless, for the large number of damage scenarios not used in training, the best sensor was S2. The HANFISes of sensor S1 and all the sensors could also predict damage locations relatively well, while the models for sensors S3 and S4 had higher errors compared to the other models. The errors for the HANFISes of LDA-FKT scores from datasets used in training were smaller compared to those of the corresponding PACC models. For DSFs from all the sensors and sensor S2, the LDA-FKT scores gave the overall best performance. These two HANFISes also led to the overall smallest errors for the damage scenarios not used in training, this time with sensor S2 coming on top.

Finally, Fig. 14 shows the error rates separately for all the tested damage scenarios. It can be seen that the variation of the indicated damage locations and extents for the damage scenarios used in training affect the performance of the different HANFISes similarly. For the varied TE locations, the attached masses of $50 \mathrm{~g}$ were more precisely localized than the $20 \mathrm{~g}$ 
masses. The opposite behaviour can be observed for the varied LE damage locations. Investigating the model accuracies for the damage locations used in training but with significantly varied damage extents reveals that all the models were confused when localizing the $10 \mathrm{~g}$ and $20 \mathrm{~g}$ attached to the TE or $90 \mathrm{~g}$ and $100 \mathrm{~g}$ on the LE. For the remaining case of mass at the TE, the performances were generally better with best results obtained for the LDA-FKT scores of sensor S2 and all the sensors. Nevertheless, the overall worst errors were found for the S3 HANFIS using the LDA-FKT scores. The PACC-based HANFIS performed for these damages similarly to the HANFIS of sensor S1 as the best model. The smallest errors for the LE damages between $10 \mathrm{~g}$ and $80 \mathrm{~g}$ were given by the LDA-FKT-based HANFIS of sensor S2 and all the sensors, followed by the PACC-based HANFIS of sensor S2. Significant differences in the localisation accuracy can be seen for the damage at the tip, where all the models had smaller errors for the $50 \mathrm{~g}$ mass than for the $20 \mathrm{~g}$ mass. Here, the best performance can be found for the LDA-FKT-based models of sensor S2 and all the sensors as well as the PACC-based HANFIS for sensor S2.

A general conclusion is that the LDA-FKT scores gave better SDL results which were also achieved with significantly less complex and smaller HANFISes. This can be explained by the fact that for SDL it is beneficial to maximize the discriminability between all the classes, in contrast to SDD where the PACCs gave better results. Furthermore, all damage locations can be considered as equally important. Although, the objective function for ranking DSFs was modified for SDL, the PACC HANFISes could not achieve the SDL performance levels of the LDA-FKT HANFISes. Thus, using more DSFs with lower sensitivity can be seen to affect adversely neuro-fuzzy models with respect to not only accuracy but also model complexity.

\section{CONCLUSIONS}

In this paper, the effects of fusing DSFs based on PACCs of acceleration signals from different sensors, transforming them with the help of the LDA-FKT and selecting the optimal DSF subsets were investigated for statistical SDD and neuro-fuzzy SDL. For both SHM tasks, the DSFs were ranked using multivariate statistical distance measures with respect to their ability to detect damage presence or distinguish between different damage locations. SDD was then conducted using the optimal DSFs subsets identified via ranking and employing statistical hypothesis testing. The more complex task of SDL was performed using hierarchical neuro-fuzzy systems, where the DSF ranking information facilitated 
incorporating the DSF selection problem into the HANFIS modelling process. These procedures were applied to the initial and transformed DSFs of accelerations from separate sensors as well as fused DSFs from all four sensors installed on a laboratory-scale WTB. The WTB was excited without contact using a simple pedestal fan for generating a wind-like airstream, while damage was simulated non-destructively by attaching different masses at three selected locations.

It was found that the optimal selections of the fused initial DSFs enabled to detect almost perfectly damage in scenarios both used and not used in training with AUCs of up to 0.95, whereas significant differences were observed when using DSFs from individual sensors with AUCs ranging between 0.59 and 0.91 for PACCs. These differences were smaller for transformed DSFs with AUCs between 0.69 and 0.86 , but the overall performance was not as good as for the selected PACCs $(\mathrm{AUC}=0.95)$. In contrast, applying the LDA-FKT to PACCs enabled to improve significantly the accuracy of damage localisation with the overall best results of $18.9 \mathrm{~cm}$ and $24.8 \mathrm{~cm}$ RMS errors for damage scenarios used and not used in training, respectively. In comparison, the best PACC HANFISes produced $30.2 \mathrm{~cm}$ and $39.8 \mathrm{~cm}$ RMS errors for the datasets used and not used in training, respectively. Furthermore, the neuro-fuzzy model complexities could be considerably reduced by using the LDA-FKT scores, e.g. the best LDA-FKT HANFIS required less than $10 \%$ of the number of parameters of the best PACC HANFIS. The performance obtained for the models based on the transformed fused DSFs was also very good as the overall second best. Therefore, it can be observed that fusing SHM data from several sensors at the feature level can be beneficial for both statistical hypothesis testing SDD and neuro-fuzzy SDL. It allows reducing computational efforts and at the same time increases the robustness of the SHM methods, as demonstrated by the assessment conducted on the damage scenarios not used in training.

Future studies will explore in more depth the effects of DSF fusion and different linear or even non-linear DSF transformations on the statistical hypothesis testing and neurofuzzy structural damage decision making, where detecting additionally damage severity and the influences of varying environmental and operational conditions will be considered.

\section{ACKNOWLEDGMENT}

Piotr Omenzetter and Simon Hoell's work on this paper within the Lloyd's Register Foundation Centre for Safety and Reliability Engineering at the University of Aberdeen was supported by Lloyd's Register Foundation. The Foundation helps to protect life and property 
by supporting engineering-related education, public engagement and the application of research.

\section{REFERENCES}

[1] C.R. Farrar, K. Worden, An introduction to structural health monitoring. Philosophical Transactions of the Royal Society A: Mathematical, Physical and Engineering Sciences 365 (2007) 303-315.

[2] Y. Deng, X. Liu, Electromagnetic imaging methods for nondestructive evaluation applications. Sensors 11 (2011) 11774-11808.

[3] S. Bagavathiappan, B.B. Lahiri, T. Saravanan, J. Philip, T. Jayakumar, Infrared thermography for condition monitoring - A review. Infrared Physics and Technology 60 (2013) 35-55.

[4] B. Ye, P. Huang, M. Fan, X. Gong, D. Hou, G. Zhang, Z. Zhou, Automatic classification of eddy current signals based on kernel methods. Nondestructive Testing and Evaluation 24 (2009) 1937.

[5] D. Kinet, P. Mégret, K.W. Goossen, L. Qiu, D. Heider, C. Caucheteur, Fiber Bragg grating sensors toward structural health monitoring in composite materials: Challenges and solutions. Sensors (Switzerland) 14 (2014) 7394-7419.

[6] D. Li, S.C.M. Ho, G. Song, L. Ren, H. Li, A review of damage detection methods for wind turbine blades. Smart Materials and Structures 24 (2015) 1-24.

[7] N.K. Mutlib, S.B. Baharom, A. El-Shafie, M.Z. Nuawi, Ultrasonic health monitoring in structural engineering: Buildings and bridges. Structural Control and Health Monitoring 23 (2016) 409422.

[8] E.P. Carden, P. Fanning, Vibration based condition monitoring: A review. Structural Health Monitoring 3 (2004) 355-377.

[9] W. Fan, P. Qiao, Vibration-based damage identification methods: A review and comparative study. Structural Health Monitoring 10 (2011) 83-111.

[10] H. Ho Thu, A. Mita, Applicability of mode-based damage assessment methods to severely damaged steel building, Proc. SPIE 8345 Sensors and Smart Structures Technologies for Civil, Mechanical, and Aerospace Systems, 2012, pp. 83453I-83453I-8. 
[11] S. Hoell, P. Omenzetter, Optimal selection of autoregressive model coefficients for early damage detectability with an application to wind turbine blades. Mechanical Systems and Signal Processing 70-71 (2016) 557-577.

[12] P. Omenzetter, O.R. de Lautour, Classification of damage in structural systems using time series analysis and supervised and unsupervised pattern recognition techniques, Sensors and Smart Structures Technologies for Civil, Mechanical, and Aerospace Systems, San Diego, USA, 2010, pp. 1-12.

[13] F.P. Kopsaftopoulos, S.D. Fassois, A functional model based statistical time series method for vibration based damage detection, localization, and magnitude estimation. Mechanical Systems and Signal Processing 39 (2013) 143-161.

[14] K.K. Nair, A.S. Kiremidjian, Time series based structural damage detection algorithm using Gaussian Mixtures Modeling. Journal of Dynamic Systems, Measurement and Control, Transactions of the ASME 129 (2007) 285-293.

[15] M. Gul, F.N. Catbas, Damage assessment with ambient vibration data using a novel time series analysis methodology. Journal of Structural Engineering 137 (2011) 1518-1526.

[16] P. Moreno-García, E. Castro, L. Romo-Melo, A. Gallego, A. Roldán, Vibration tests in CFRP plates for damage detection via non-parametric signal analysis. Shock and Vibration 19 (2012) $857-865$.

[17] E. Simon Carbajo, R. Simon Carbajo, C. Mc Goldrick, B. Basu, ASDAH: An automated structural change detection algorithm based on the Hilbert-Huang transform. Mechanical Systems and Signal Processing 47 (2014) 78-93.

[18] I. Trendafilova, R. Palazzetti, A. Zucchelli, Damage assessment based on general signal correlation. Application for delamination diagnosis in composite structures. European Journal of Mechanics, A/Solids 49 (2015) 197-204.

[19] J.P. Amezquita-Sanchez, H. Adeli, Signal Processing Techniques for Vibration-Based Health Monitoring of Smart Structures. Archives of Computational Methods in Engineering 23 (2016) $1-15$.

[20] P.R. Kumar, T. Oshima, T. Yamazaki, S. Mikami, Y. Miyamouri, Detection and localization of small damages in a real bridge by local excitation using piezoelectric actuators. Journal of Civil Structural Health Monitoring 2 (2012) 97-108. 
[21] S. Beskhyroun, T. Oshima, S. Mikami, Y. Miyamori, Assessment of vibration-based damage identification techniques using localized excitation source. Journal of Civil Structural Health Monitoring 3 (2013) 207-223.

[22] H. Zheng, A. Mita, Localized damage detection of structures subject to multiple ambient excitations using two distance measures for autoregressive models. Structural Health Monitoring 8 (2009) 207-222.

[23] Y. An, B. Li, J. Ou, An algorithm for damage localization in steel truss structures: Numerical simulation and experimental validation. Journal of Intelligent Material Systems and Structures 24 (2013) 1683-1698.

[24] G. Liu, Z. Mao, M. Todd, Z. Huang, Localization of nonlinear damage using state-space-based predictions under stochastic excitation. Smart Materials and Structures 23 (2014) 1-14.

[25] M.M. Alamdari, B. Samali, J. Li, Damage localization based on symbolic time series analysis. Structural Control and Health Monitoring 22 (2015) 374-393.

[26] G. Manson, R.J. Barthorpe, Advanced feature selection for simplified pattern recognition within the damage identification framework. Shock and Vibration 17 (2010) 589-599.

[27] D. Bernal, Flexibility-based damage localization from stochastic realization results. Journal of Engineering Mechanics 132 (2006) 651-658.

[28] M. Döhler, L. Marin, D. Bernal, L. Mevel, Statistical decision making for damage localization with stochastic load vectors. Mechanical Systems and Signal Processing 39 (2013) 426-440.

[29] M.I. Friswell, Damage identification using inverse methods. Philosophical Transactions of the Royal Society A: Mathematical, Physical and Engineering Sciences 365 (2007) 393-410.

[30] M.M. Saada, M.H. Arafa, A.O. Nassef, Finite element model updating approach to damage identification in beams using particle swarm optimization. Engineering Optimization 45 (2013) 677-696.

[31] F. Shabbir, P. Omenzetter, Particle swarm optimization with sequential niche technique for dynamic finite element model updating. Computer-Aided Civil and Infrastructure Engineering 30 (2015) 359-375. 
[32] B. Titurus, M.I. Friswell, Regularization in model updating. International Journal for Numerical Methods in Engineering 75 (2008) 440-478.

[33] K. Worden, W.J. Staszewski, J.J. Hensman, Natural computing for mechanical systems research: A tutorial overview. Mechanical Systems and Signal Processing 25 (2011) 4-111.

[34] Q. Zhou, H. Zhou, Q. Zhou, F. Yang, L. Luo, Structure damage detection based on random forest recursive feature elimination. Mechanical Systems and Signal Processing 46 (2014) 82-90.

[35] S.B. Satpal, A. Guha, S. Banerjee, Damage identification in aluminium beams using support vector machine: Numerical and experimental studies. Structural Control and Health Monitoring 23 (2016) 446-457.

[36] D.A. Tibaduiza, M.A. Torres-Arredondo, L.E. Mujica, J. Rodellar, C.P. Fritzen, A study of two unsupervised data driven statistical methodologies for detecting and classifying damages in structural health monitoring. Mechanical Systems and Signal Processing 41 (2013) 467-484.

[37] R.A. Osornio-Rios, J.P. Amezquita-Sanchez, R.J. Romero-Troncoso, A. Garcia-Perez, MUSICANN Analysis for Locating Structural Damages in a Truss-Type Structure by Means of Vibrations. Computer-Aided Civil and Infrastructure Engineering 27 (2012) 687-698.

[38] S.J.S. Hakim, H. Abdul Razak, Frequency response function-based structural damage identification using artificial neural networks - A review. Research Journal of Applied Sciences, Engineering and Technology 7 (2014) 1750-1764.

[39] C. Park, J. Tang, Y. Ding, Aggressive data reduction for damage detection in structural health monitoring. Structural Health Monitoring 9 (2010) 59-74.

[40] E. Zugasti, L.E. Mujica, J. Anduaga, F. Martinez, Feature selection - Extraction methods based on PCA and mutual information to improve damage detection problem in offshore wind turbines. Key Engineering Materials 569-570 (2013) 620-627.

[41] I.T. Jolliffe, Principal Component Analysis, Springer, New York, USA, 2002.

[42] A.M. Yan, G. Kerschen, P. De Boe, J. Golinval, Structural damage diagnosis under varying environmental conditions - Part II: Local PCA for non-linear cases. Mechanical Systems and Signal Processing 19 (2005) 865-880. 
[43] E.J. Cross, G. Manson, K. Worden, S.G. Pierce, Features for damage detection with insensitivity to environmental and operational variations. Proceedings of the Royal Society A: Mathematical, Physical and Engineering Sciences 468 (2012) 4098-4122.

[44] R.P. Bandara, T.H. Chan, D.P. Thambiratnam, Structural damage detection method using frequency response functions. Structural Health Monitoring 13 (2014) 418-429.

[45] J. Kullaa, Damage detection of the Z24 bridge using control charts. Mechanical Systems and Signal Processing 17 (2003) 163-170.

[46] M.R. Hernandez-Garcia, S.F. Masri, Application of statistical monitoring using latent-variable techniques for detection of faults in sensor networks. Journal of Intelligent Material Systems and Structures 25 (2014) 121-136.

[47] M.A. Torres-Arredondo, J. Sierra Pérez, D.A. Tibaduiza, M. McGugan, J. Rodellar, C.P. Fritzen, Signal-based nonlinear modelling for damage assessment under variable temperature conditions by means of acousto-ultrasonics. Structural Control and Health Monitoring 22 (2015) 11031118.

[48] Y. Liu, A. Chattopadhyay, Low-velocity impact damage monitoring of a sandwich composite wing. Journal of Intelligent Material Systems and Structures 24 (2013) 2074-2083.

[49] N. Dervilis, I. Antoniadou, E.J. Cross, K. Worden, A non-linear manifold strategy for SHM approaches. Strain 51 (2015) 324-331.

[50] S. Prabhu, S. Atamturktur, Feature assimilation for vibration based damage detection. Journal of Testing and Evaluation 41 (2013) 1-11.

[51] K. Fukunaga, W.L.G. Koontz, Application of the Karhunen-Loéve Expansion to Feature Selection and Ordering. IEEE Transactions on Computers C-19 (1970) 311-318.

[52] X. Huo, A statistical analysis of Fukunaga-Koontz transform. IEEE Signal Processing Letters 11 (2004) 123-126.

[53] S. Zhang, T. Sim, Discriminant subspace analysis: A Fukunaga-Koontz approach. IEEE Transactions on Pattern Analysis and Machine Intelligence 29 (2007) 1732-1745.

[54] J.S.R. Jang, ANFIS: adaptive-network-based fuzzy inference system. IEEE Transactions on Systems, Man, and Cybernetics 23 (1993) 665-685. 
[55] J.S.R. Jang, C.T. Sun, E. Mizutani, Neuro-Fuzzy and Soft Computing: A Computational Approach to Learning and Machine Intelligence, Prentice-Hall, Upper Saddle River, USA, 1997.

[56] Y. Lei, Z. He, Y. Zi, Q. Hu, Fault diagnosis of rotating machinery based on multiple ANFIS combination with GAs. Mechanical Systems and Signal Processing 21 (2007) 2280-2294.

[57] M. Brown, K.M. Bossley, D.J. Mills, C.J. Harris, High dimensional neurofuzzy systems: overcoming the curse of dimensionality, International Joint Conference of the Fourth IEEE International Conference on Fuzzy Systems and the Second International Fuzzy Engineering Symposium, Yokohama, Japan, 1995, pp. 2139-2146.

[58] S.M. Kay, Modern Spectral Estimation: Theory and Application, Prentice Hall, Upper Saddle River, USA, 1988.

[59] United Nations Framework Convention on Climate Change, Adoption of the Paris Agreement. (2015) 1-32.

[60] M.I. Blanco, The economics of wind energy. Renewable and Sustainable Energy Reviews 13 (2009) 1372-1382.

[61] J.D. Cryer, K. Chan, Time Series Analysis with Applications in R, Springer Science+Business Media, New York, USA, 2008.

[62] G.E.P. Box, G.M. Jenkins, G.C. Reinsel, Time Series Analysis Forecasting and Control, John Wiley \& Sons, Hoboken, USA, 2008.

[63] M.H. Quenouille, Approximate Tests of Correlation in Time-Series. Journal of the Royal Statistical Society: Series B (Methodological) 11 (1949) 68-84.

[64] D.C. Montgomery, Introduction to Statistical Quality Control, John Wiley \& Sons, New York, USA, 1997.

[65] D. Rasch, J. Pilz, R. Verdooren, A. Gebhardt, Optimal Experimental Design with R, Taylor \& Francis, Boca Raton, USA, 2011.

[66] C.M. Bishop, Pattern Recognition and Machine Learning, Springer Science + Business Media, New York, USA, 2006.

[67] K. Fukunaga, Introduction to Statistical Pattern Recognition, Academic Press, San Diego, USA, 1990. 
[68] R. Huang, Q. Liu, H. Lu, S. Ma, Solving the small sample size problem of LDA, 16th International Conference on Pattern Recognition, Quebec, Canada, 2002, pp. 29-32.

[69] A.C. Rencher, Multivariate Statistical Inference and Applications, John Wiley \& Sons, New York, USA, 1998.

[70] S. Arlot, A. Celisse, A survey of cross-validation procedures for model selection. Statistics Surveys 4 (2010) 40-79.

[71] S. Ataya, M.M.Z. Ahmed, Damages of wind turbine blade trailing edge: Forms, location, and root causes. Engineering Failure Analysis 35 (2013) 480-488.

[72] J.C. Marín, A. Barroso, F. París, J. Cañas, Study of fatigue damage in wind turbine blades. Engineering Failure Analysis 16 (2009) 656-668.

[73] F.M. Jensen, B.G. Falzon, J. Ankersen, H. Stang, Structural testing and numerical simulation of a 34 m composite wind turbine blade. Composite Structures 76 (2006) 52-61.

[74] C.C. Ciang, J.R. Lee, H.J. Bang, Structural health monitoring for a wind turbine system: A review of damage detection methods. Measurement Science and Technology 19 (2008) 1-20.

[75] W.J. Krzanowski, D.J. Hand, ROC Curves for Continuous Data, Taylor \& Francis, Boca Raton, USA, 2009. 
Baseline Phase

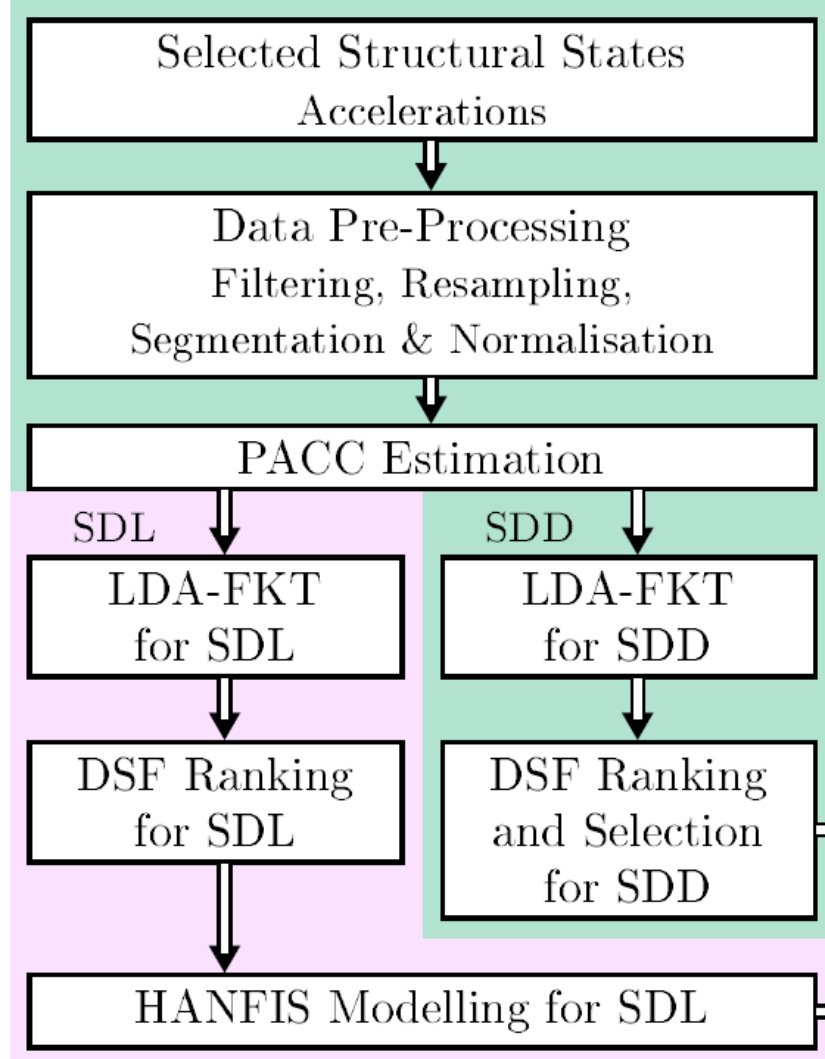

* If established as beneficial in Baseline Phase

Operational Phase

Current State

Accelerations

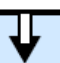

Data Pre-Processing

Filtering, Resampling,

Segmentation \& Normalisation

4

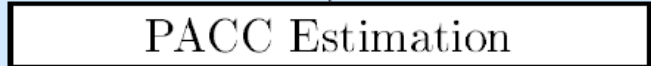

LDA-FKT for SDD*

Statistical SDD

Selected (Transformed*) DSFs

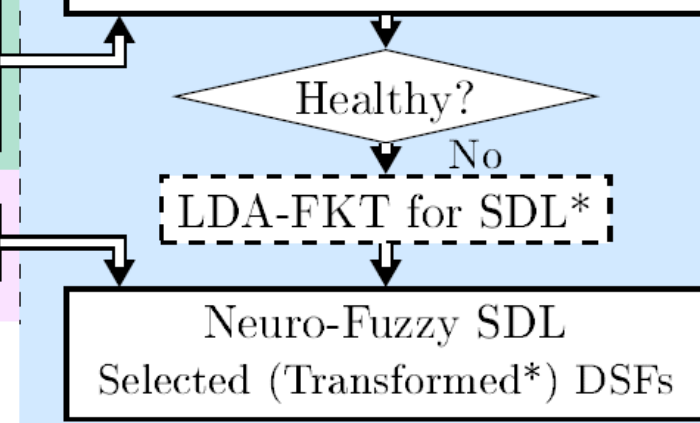

Figure 1 Methodology for SDD and SDL 


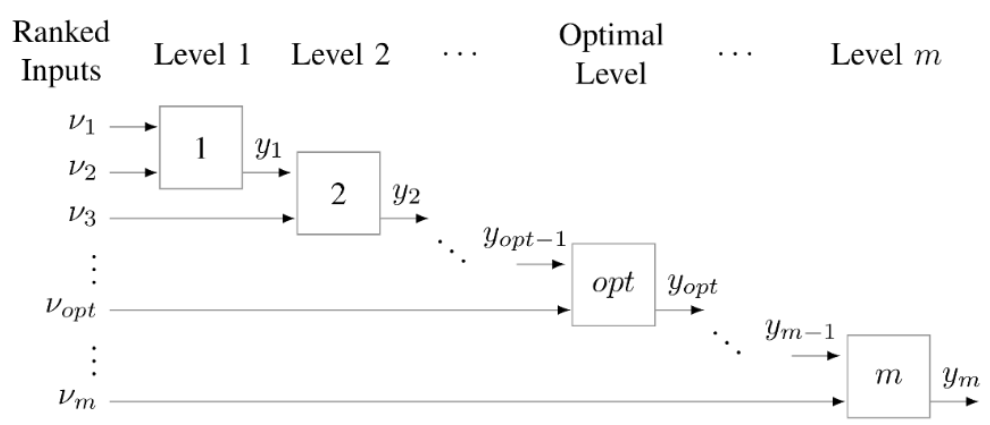

Figure 2 HANFIS architecture (each square indicates an individual ANFIS shown in Fig. 3) 


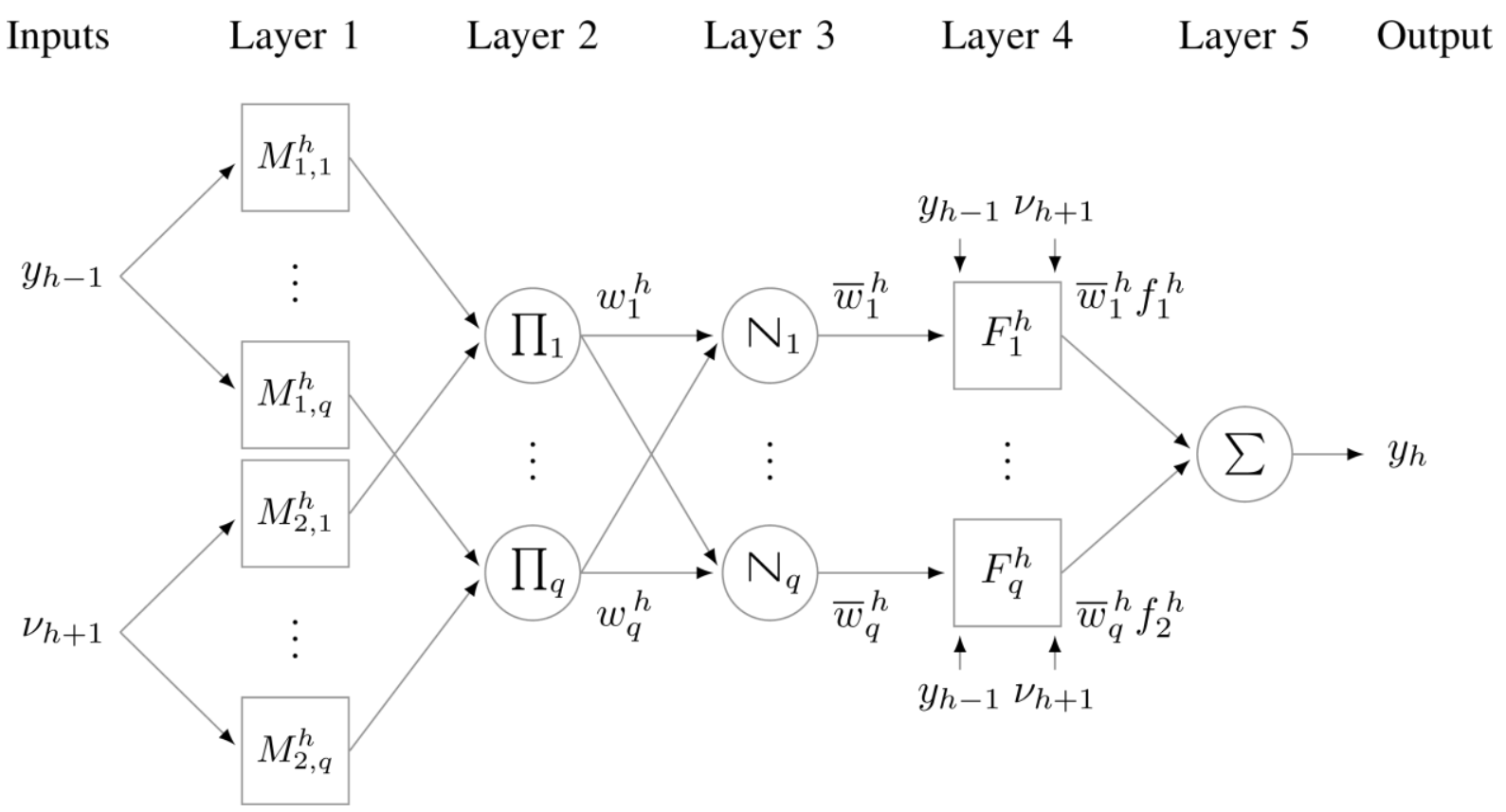

Figure 3 Architecture of the $h$-th individual ANFIS (full HANFIS architecture is shown in Fig. 2) 


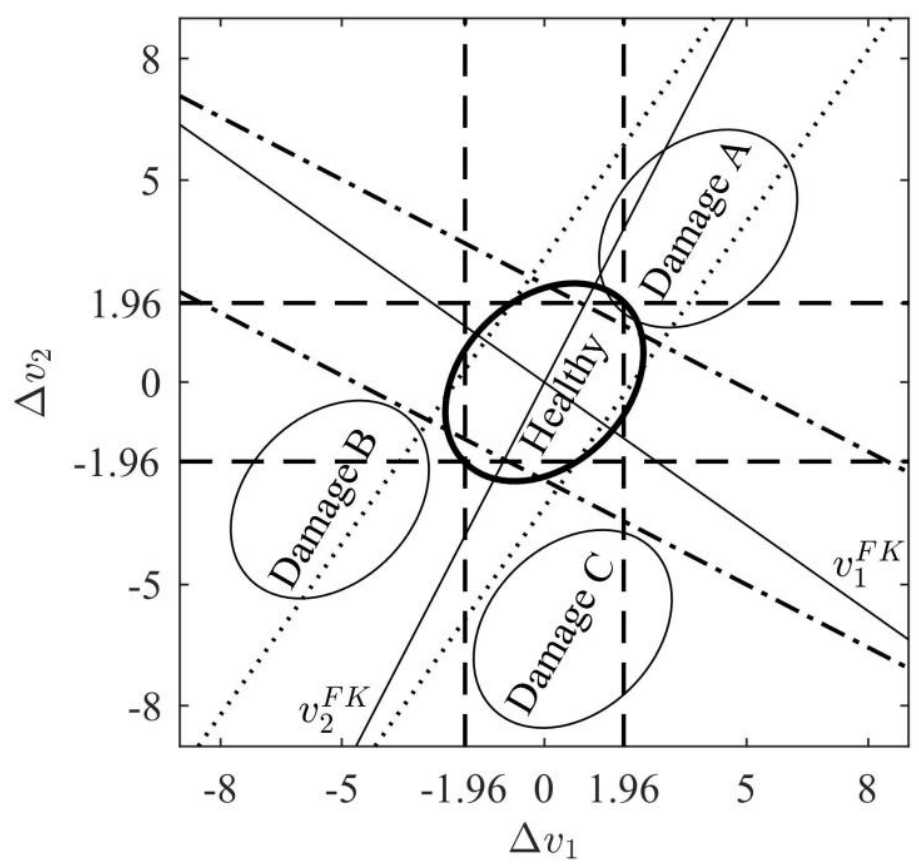

Hypothesis testing thresholds:

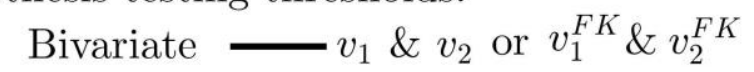

a)

Univariate $--v_{1}$ or $v_{2} \quad \cdots \cdots \cdot v_{1}^{F K} \quad-\cdot-\cdot v_{2}^{F K}$

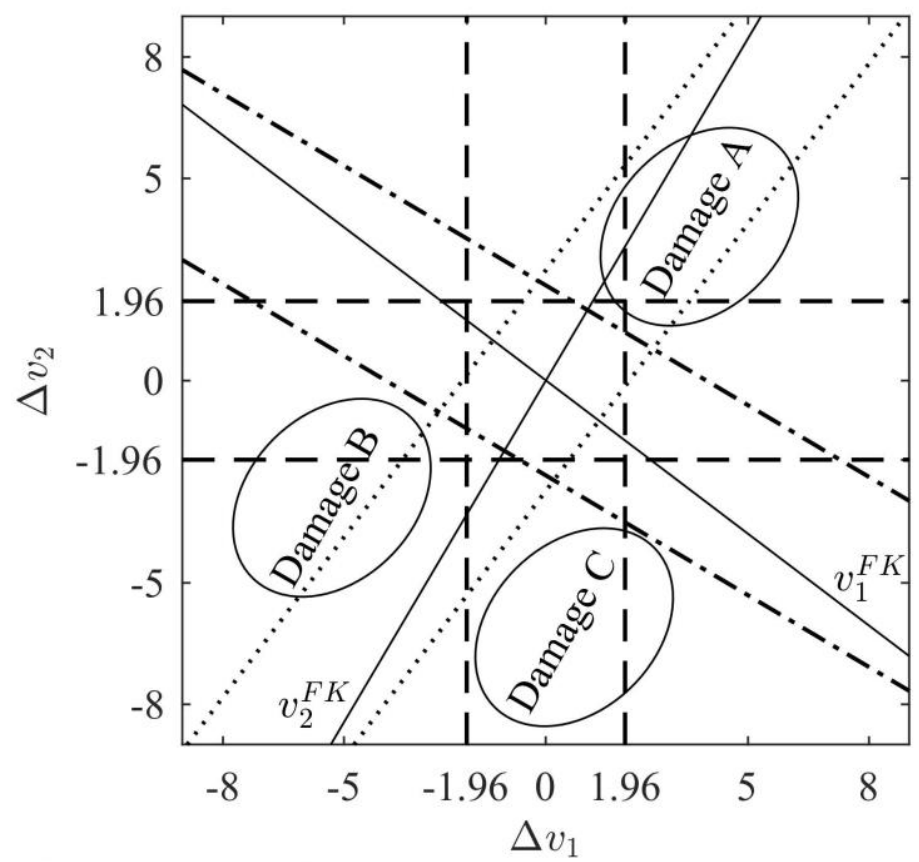

Hypothesis testing thresholds:

b)

Univariate $--v_{1}$ or $v_{2} \quad \cdots \cdots \cdot v_{1}^{F K} \quad-\cdot-\cdot v_{2}^{F K}$

Figure 4 Case 1 of bivariate problem illustration: a) SDD, and b) SDL 


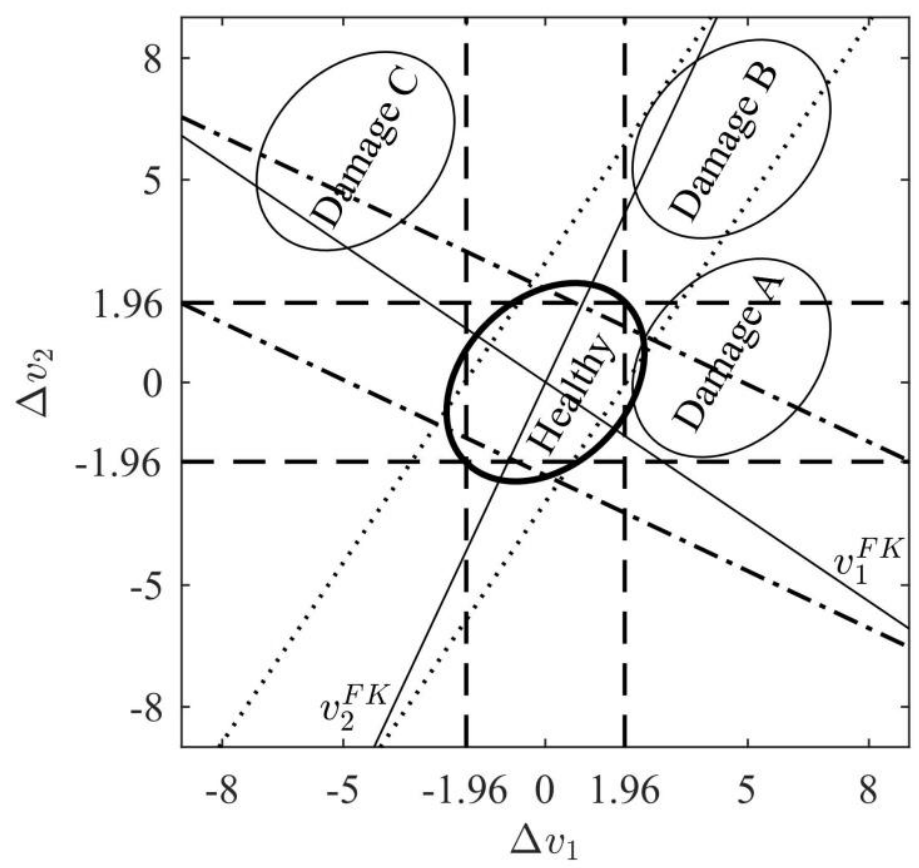

Hypothesis testing thresholds:

Bivariate $-v_{1} \& v_{2}$ or $v_{1}^{F K} \& v_{2}^{F K}$

a)

Univariate $--v_{1}$ or $v_{2} \quad \cdots \cdots \cdots v_{1}^{F K} \quad-\cdot-\cdot v_{2}^{F K}$

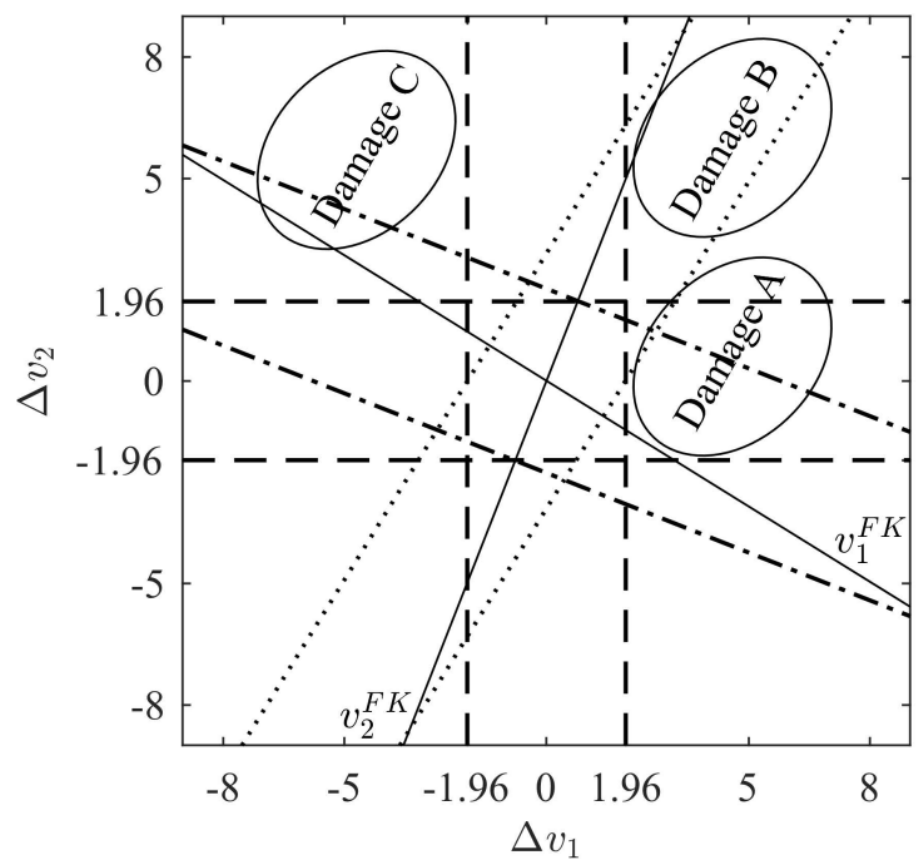

Hypothesis testing thresholds:

b)

Univariate $--v_{1}$ or $v_{2}$ $v_{1}^{F K}-\cdot-\cdot v_{2}^{F K}$

Figure 5 Case 2 of bivariate problem illustration: a) SDD, and b) SDL 


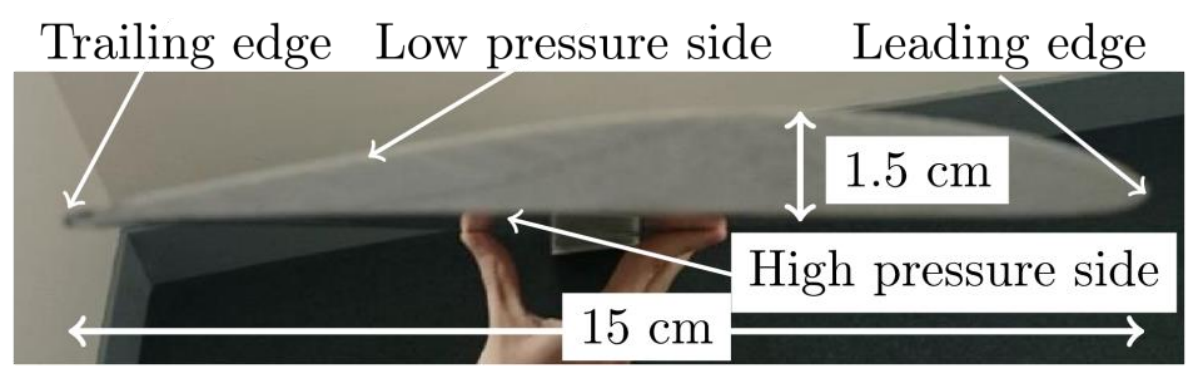

Figure 6 Experimental wind turbine blade cross-section 


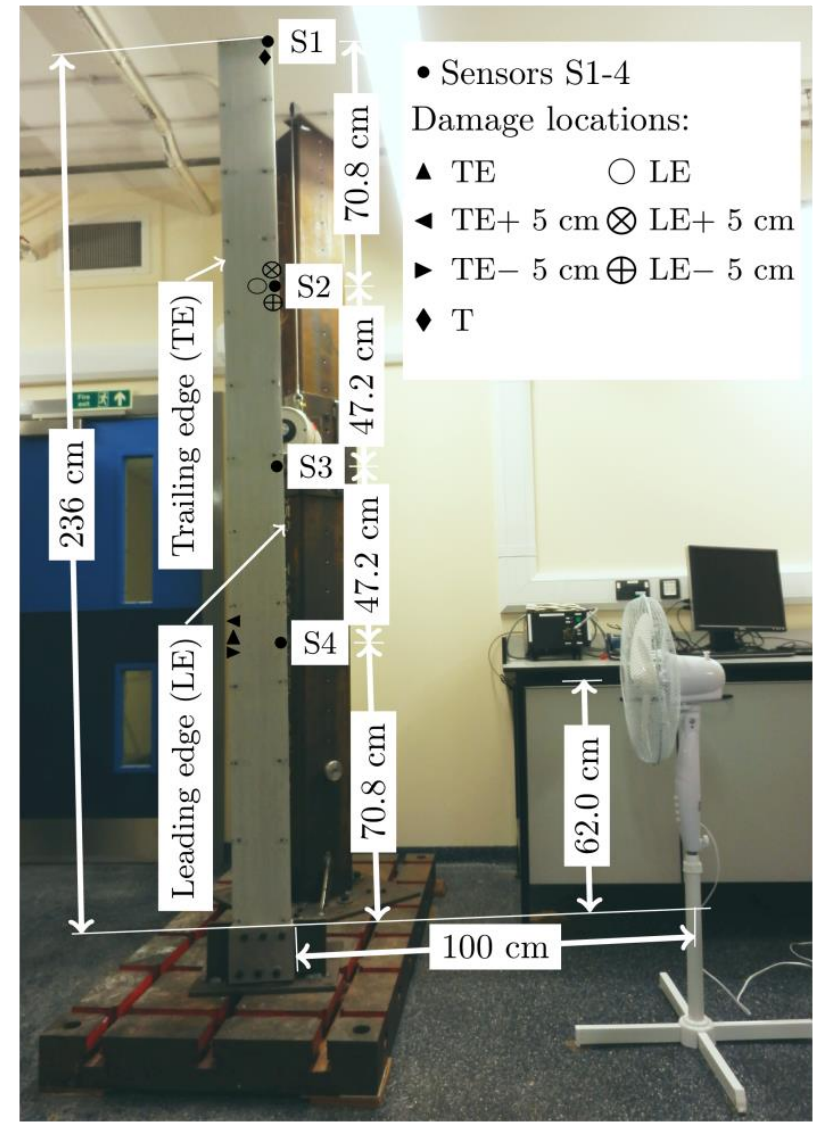

Figure 7 Experimental configuration 


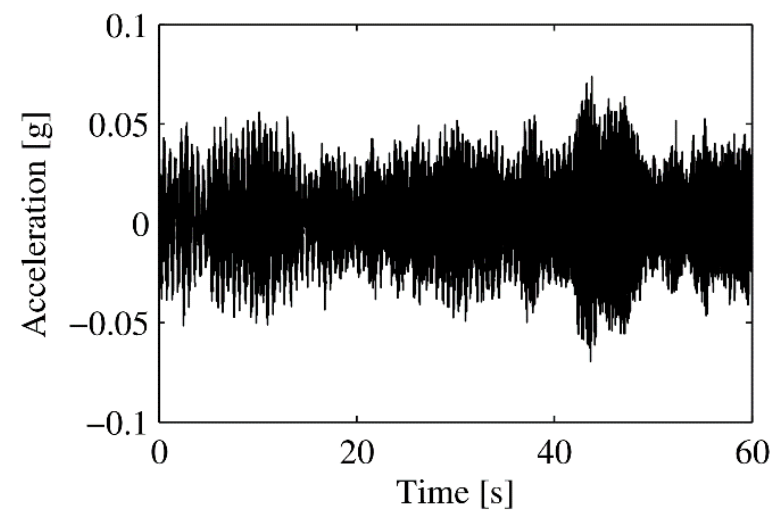

Figure 8 Example of acceleration time series of the healthy WTB at sensor S1 
PACF: FF ranking for SDD

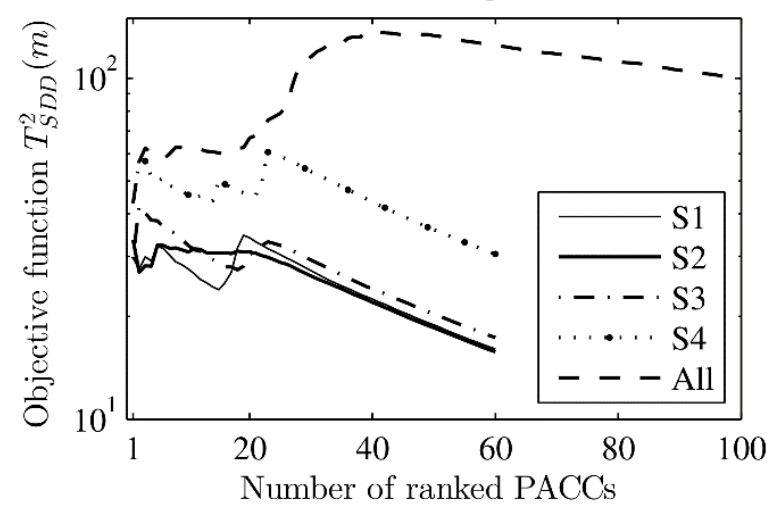

a)

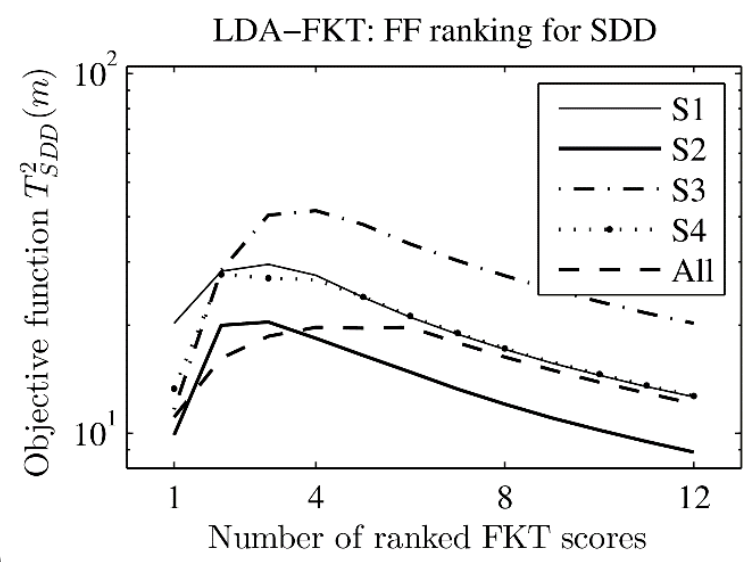

b)

Figure 9 Objective function for SDD, $T_{S D D}^{2}$ (m) (Eq. (26)), for individual sensors S1-S4 and all the sensors, and increasing numbers of ranked DSFs: a) PACCs, and b) LDA-FKT scores 


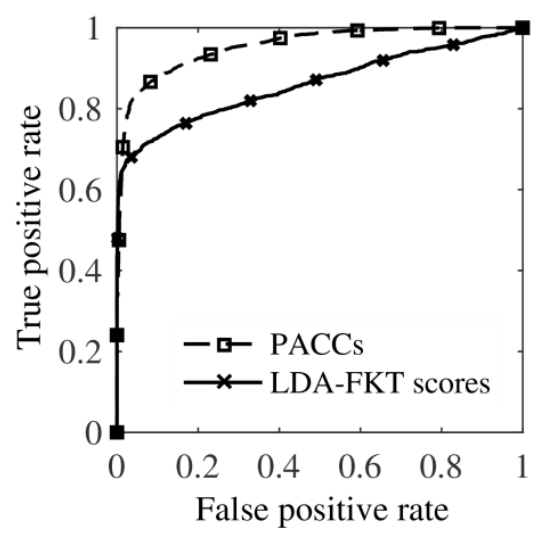

Figure 10 Receiver operating characteristic curve for selected PACCs and LDA-FKT scores from all the sensors obtained for previously unseen data from scenarios used in training 


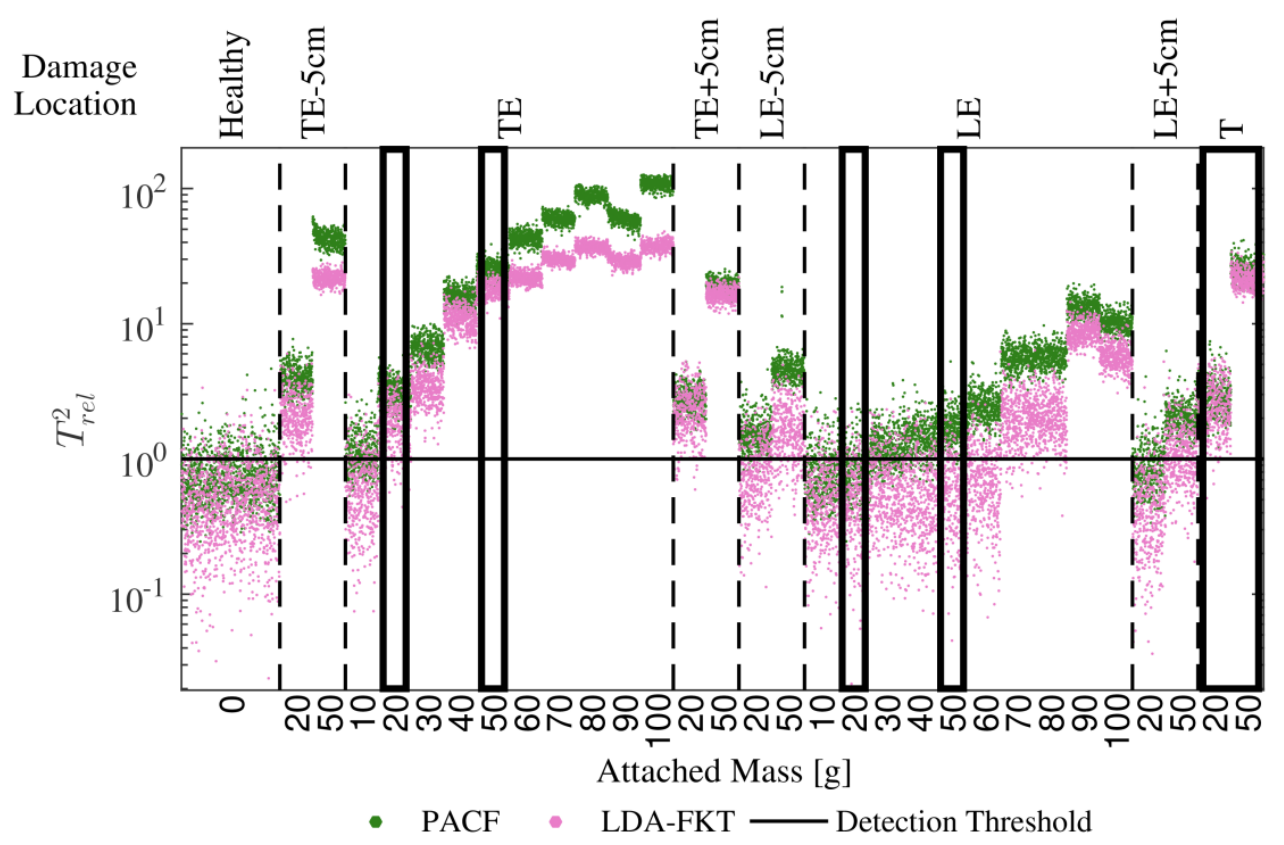

Figure 11 Relative statistical distances, $T_{\text {rel }}^{2}$, (Eq. (25)) for single samples of selected PACCs and LDA-FKT scores from all the sensors obtained for previously unseen healthy and damage scenario datasets used (bold frames) and not used in training (no frames) 


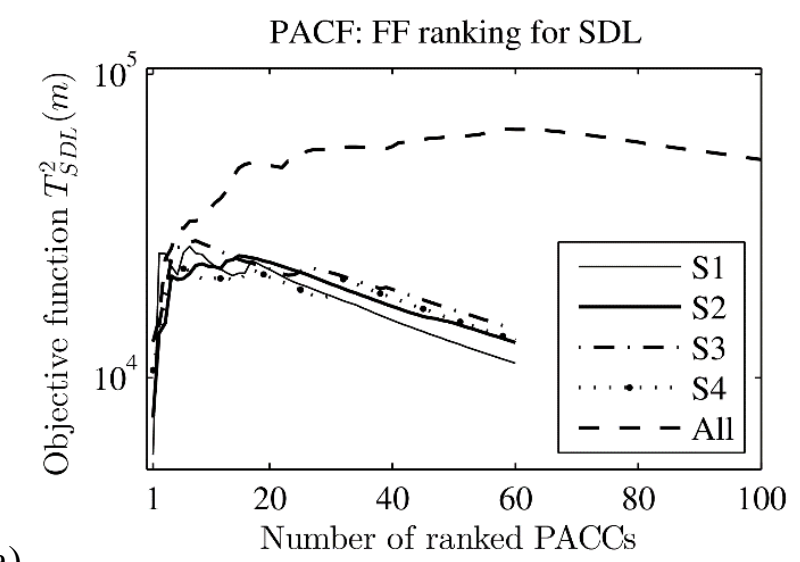

a)

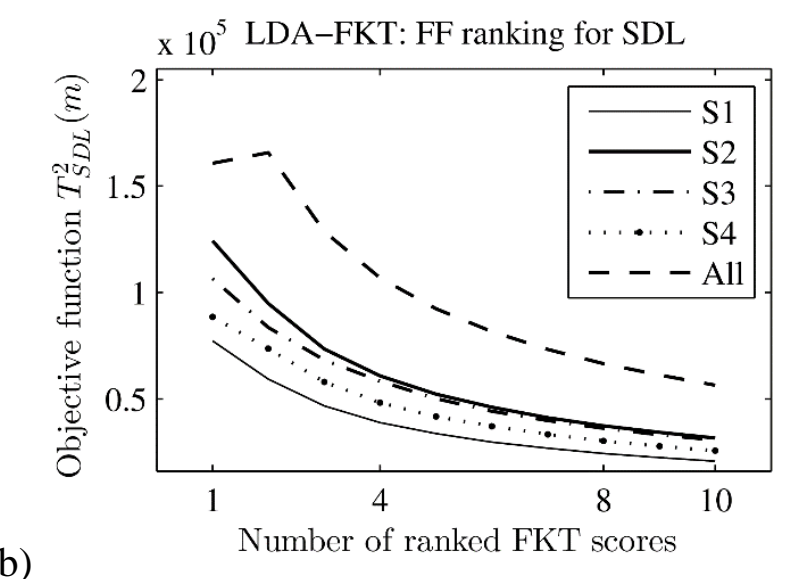

Figure 12 Objective function for SDL, $T_{S D L}^{2}(\mathrm{~m})$ (Eq. (27)), for individual sensors S1-S4 and all the sensors, and increasing numbers of ranked DSFs: a) PACCs, and b) LDA-FKT scores 
PACF: HANFIS for SDL

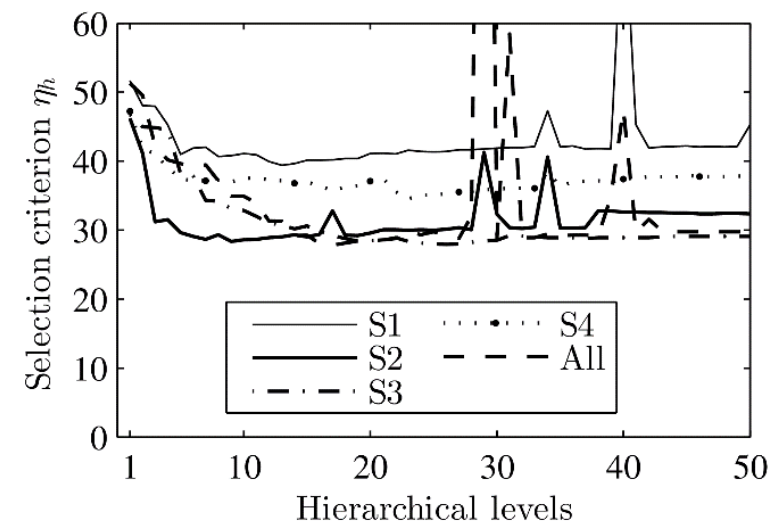

a)

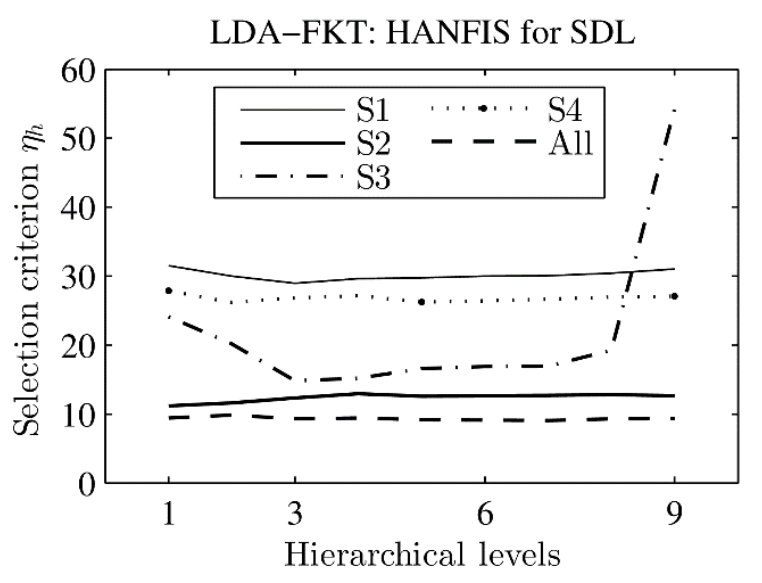

b)

Figure 13 Model selection criterion $\eta_{h}$ (Eq. (29)) for individual sensors S1-S4 and all the sensors, and increasing numbers of hierarchical levels: a) PACF HANFIS, and b) LDA-FKT HANFIS 


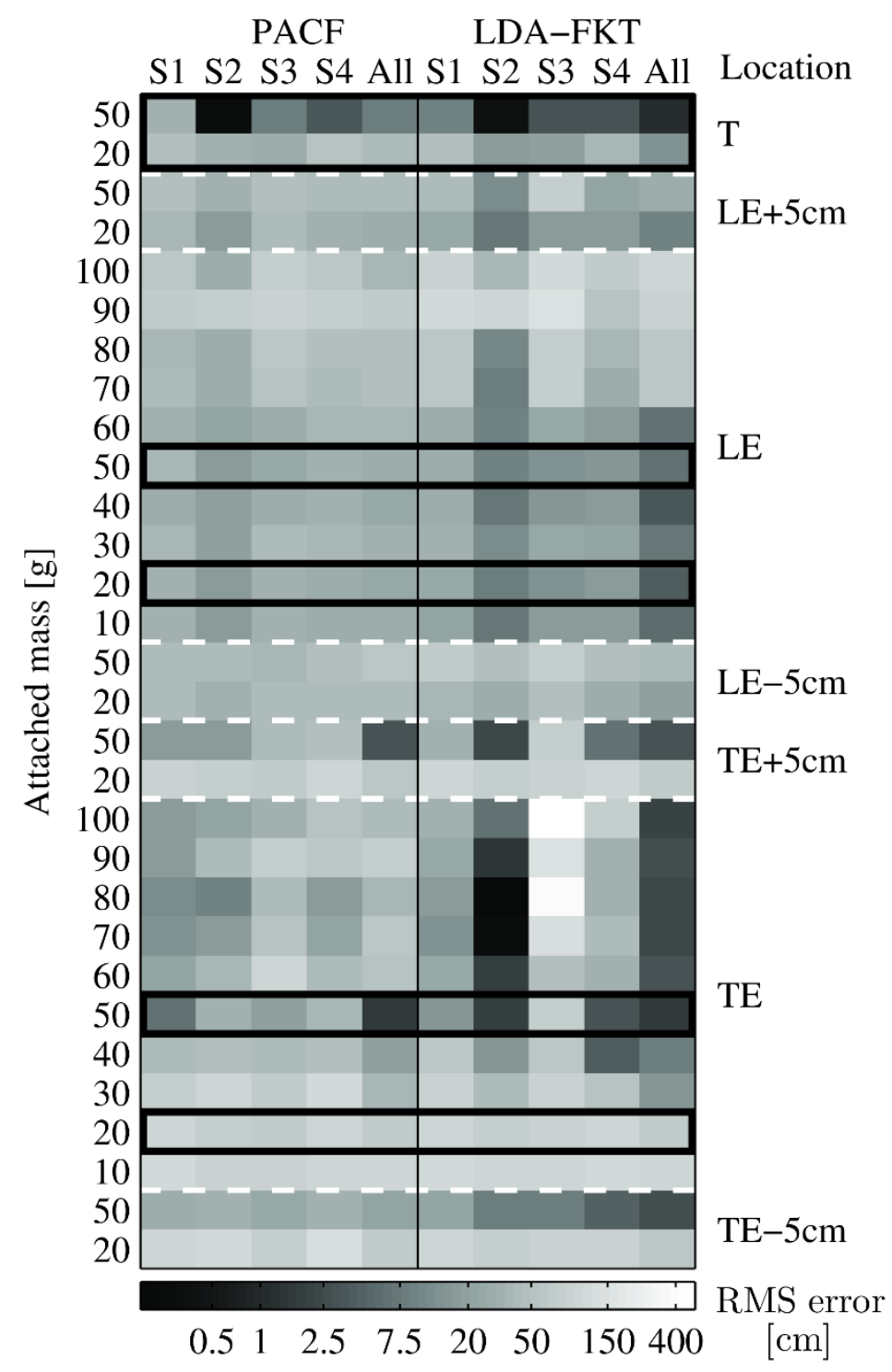

Figure 14 Root mean square error of HANFIS models for previously unseen damage scenario datasets used (bold frames) and not used in training (no frames) (colours change with log scale; TE- $5 \mathrm{~cm}, \mathrm{TE}, \mathrm{TE}+5 \mathrm{~cm}, \mathrm{LE}-5 \mathrm{~cm}, \mathrm{LE}, \mathrm{LE}+5 \mathrm{~cm}$ and $\mathrm{T}$ refer to damage locations indicated in Fig. 7) 
Table 1

Damage scenarios

\begin{tabular}{ccccc} 
Position & Name $^{*}$ & $\begin{array}{c}\text { Distance } \\
\text { from root } \\
{[\mathrm{cm}]}\end{array}$ & Training & Testing \\
\hline $\begin{array}{c}\text { Trailing } \\
\text { edge } \\
(\mathrm{TE})\end{array}$ & TE-5cm & 67.3 & - & 20,50 \\
\hline TE $+5 \mathrm{~cm}$ & 77.3 & - & 20,50 \\
\hline $\begin{array}{c}\text { Leading } \\
\text { edge } \\
(\mathrm{LE})\end{array}$ & LE-5cm & 160.2 & - & 2,5 \\
\hline Tip (T) & LE $+5 \mathrm{~cm}$ & 170.2 & - & $10-100^{* * *}$ \\
\hline
\end{tabular}

${ }^{*}$ TE- $5 \mathrm{~cm}, \mathrm{TE}, \mathrm{TE}+5 \mathrm{~cm}, \mathrm{LE}-5 \mathrm{~cm}, \mathrm{LE}, \mathrm{L}+5 \mathrm{~cm}$ and $\mathrm{T}$ refer to damage locations indicated in Fig. 7

** $10 \mathrm{~g}$ increments 
Table 2

AUCs for SDD for previously unseen data used and not used in training

\begin{tabular}{cccc}
\multirow{2}{*}{ DSF } & Sensor & \multicolumn{2}{c}{ AUC } \\
& & Data used in training & Data not used in training \\
\hline \multirow{4}{*}{ PACF } & S1 & 0.84 & 0.84 \\
& S2 & 0.59 & 0.59 \\
& S3 & 0.75 & 0.75 \\
& S4 & 0.91 & 0.91 \\
LDA-FKT & S1l & 0.95 & 0.95 \\
\hline \multirow{5}{*}{} & S2 & 0.78 & 0.78 \\
& S3 & 0.69 & 0.69 \\
& S4 & 0.86 & 0.86 \\
& All & 0.86 & 0.86 \\
& & 0.86 & 0.86
\end{tabular}


Table 3

HANFIS level selection for SDL and corresponding numbers of model parameters

\begin{tabular}{cccc} 
DSF & Sensor & Level & $\begin{array}{c}\text { Total number of } \\
\text { parameters }\end{array}$ \\
\hline \multirow{4}{*}{ PACF } & S1 & 13 & 1,302 \\
& S2 & 9 & 819 \\
& S3 & 25 & 2,093 \\
& S4 & 23 & 1,932 \\
LDA-FKT & All & 17 & 1,561 \\
\hline \multirow{5}{*}{ S1 } & S2 & 3 & 371 \\
& S3 & 3 & 63 \\
& S4 & 2 & 231 \\
& All & 3 & 119 \\
& & & 210
\end{tabular}


Table 4

Root mean square errors for SDL for previously unseen data used and not used in training

\begin{tabular}{|c|c|c|c|}
\hline \multirow{2}{*}{ DSF } & \multirow[t]{2}{*}{ Sensor } & \multicolumn{2}{|c|}{ RMS error $[\mathrm{cm}]$} \\
\hline & & Data used in training & Data not used in training \\
\hline \multirow{5}{*}{ PACF } & S1 & 40.9 & 43.3 \\
\hline & $\mathrm{S} 2$ & 30.2 & 39.8 \\
\hline & S3 & 30.0 & 52.7 \\
\hline & S4 & 43.2 & 52.4 \\
\hline & All & 29.0 & 44.3 \\
\hline \multirow{5}{*}{ LDA-FKT } & $\mathrm{S} 1$ & 36.9 & 50.7 \\
\hline & $\mathrm{S} 2$ & 18.9 & 24.8 \\
\hline & S3 & 35.9 & 101.5 \\
\hline & S4 & 28.4 & 39.4 \\
\hline & All & 15.7 & 28.6 \\
\hline
\end{tabular}

